\title{
Retrospective-Cost Adaptive Control of Uncertain Hammerstein-Wiener Systems with Memoryless and Hysteretic Nonlinearities
}

\author{
Mohammad Al Janaideh*, Jin Yan †, Anthony M. D’Amato $\ddagger$ and Dennis S. Bernstein ${ }^{\S}$ \\ University of Michigan, 1320 Beal Ave., Ann Arbor, MI 48109
}

\begin{abstract}
We apply retrospective cost adaptive control (RCAC) with auxiliary nonlinearities to a command-following problem for uncertain Hammerstein-Wiener systems with memoryless and hysteretic nonlinearities. The only required modeling information of the linear plant is a single Markov parameter. To account for the nonlinearities, RCAC uses auxiliary nonlinearities that reflect the monotonicity properties of the nonlinearity. Various memoryless nonlinearities such as deadband, cubic, and saturation are considered. The hysteresis nonlinearity is modeled using the Prandtl-Ishlinskii model.
\end{abstract}

\section{Introduction}

The simplest extension of linear systems to nonlinear systems is to cascade the linear plant with input and output nonlinearities. The presence of an input nonlinearity constitutes a Hammerstein system, while the output nonlinearity constitutes a Wiener system. The presence of both input and output nonlinearities constitutes a Hammerstein-Wiener $(H W)$ system..$^{1-4}$

Hammerstein systems model linearized dynamics with actuator nonlinearities, whereas Wiener systems model linearized dynamics with sensor nonlinearities. These nonlinearities typically arise from properties of the hardware realization of sensors and actuators, for example, mechanical linkages or electromechanical devices. These nonlinearities may be memoryless, such as saturation and deadband, or they may have internal dynamics due to smart materials that exhibit hysteresis.

If the nonlinearities are known, then in some cases they can be inverted in software, and the resulting system is effectively linear. However, a saturation input nonlinearity limits the range of the input signal, while a saturation output nonlinearity limits the ability to observe the response of the system. Finally, both input and output nonlinearities distort the input-output behavior of the system, producing spurious harmonics.

Control of Hammerstein-Wiener systems is more challenging when the input and output nonlinearities as well as the linear plant dynamics are uncertain. Uncertainty may arise from imperfect identification or unexpected changes to the system during operation. Although robust control methods may be effective, ${ }^{5,6}$ these techniques require characterization of the uncertainty and sacrifice performance for reliability.

\footnotetext{
*Assistant Professor, Department of Mechatronics Engineering, The University of Jordan, 11942 Amman, Jordan

$\dagger$ Graduate Student, Aerospace Engineering Department

${ }^{\ddagger}$ NASA GSRP Fellow, Aerospace Engineering Department

$\S$ Professor, Aerospace Engineering Department
} 


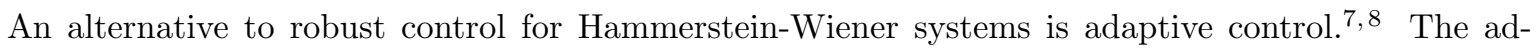
vantage of adaptive control relative to robust control is the ability of the controller to tune itself to the actual plant despite modeling errors and unexpected changes. Although some modeling information about the nonlinearities and linear dynamics may be required, the need to model the system before operation may be reduced.

In the present paper we apply retrospective cost adaptive control (RCAC) to Hammerstein-Wiener systems with uncertain linear dynamics and uncertain input and output nonlinearities, which may be either memoryless or hysteretic. Details on RCAC for linear systems are given in. ${ }^{9-12}$ The present paper extends the application of RCAC to Hammerstein systems in. ${ }^{13,15}$

$\mathrm{In}^{13,15}$ the adaptive control is instantaneously linear, and thus converges to a linear time-invariant controller. Consequently, RCAC seeks a linear controller that is effective in reducing the distortion due to the input and output nonlinearities. Nonlinear controller structures that further reduce this distortion are considered in. ${ }^{16}$

\section{ADAPTIVE CONTROL FOR THE HAMMERSTEIN-WIENER COMMAND-FOLLOWING PROBLEM}

Consider the Hammerstein-Wiener command-following problem

$$
\begin{aligned}
u_{0}(k) & =\mathcal{N}_{H}(u(k)), \\
x(k+1) & =A x(k)+B u_{0}(k)+D_{1} w(k), \\
y_{0}(k) & =E_{1} x(k)+D_{2} w(k), \\
y(k) & =\mathcal{N}_{W}\left(y_{0}(k)\right), \\
z(k) & =r(k)-y(k),
\end{aligned}
$$

where $x(k) \in \mathbb{R}^{n}$ is the state, $r(k) \in \mathbb{R}^{l_{r}}$ is the command, $u(k) \in \mathbb{R}^{l_{u}}$ is the control, $w(k) \in \mathbb{R}^{l_{w}}$ is the exogenous signal, $\mathcal{N}_{H}: \mathbb{R} \rightarrow \mathbb{R}$ is the input nonlinearity, $\mathcal{N}_{W}: \mathbb{R} \rightarrow \mathbb{R}$ is the output nonlinearity, and $z(k) \in \mathbb{R}^{l_{z}}$ is the command-following error. We assume that $G$ is uncertain except for a limited number of Markov parameters. The input nonlinearity $\mathcal{N}_{H}$ and output nonlinearity $\mathcal{N}_{W}$ are also uncertain. We assume that the output nonlinearity $\mathcal{N}_{W}$ is increasing, while the input nonlinearity $\mathcal{N}_{H}$ has known monotonicity. The intermediate signals $v(k)$ and $y_{0}(k)$ are assumed to be unknown. A block diagram for (1)-(5) is shown in Figure 1. We apply RCAC to the Hammerstein-Wiener system in order to have the output $y$ follow the command signal $r$. The auxiliary nonlinearities $\mathcal{N}_{1}$ and $\mathcal{N}_{2}$ are discussed in Section III.C.

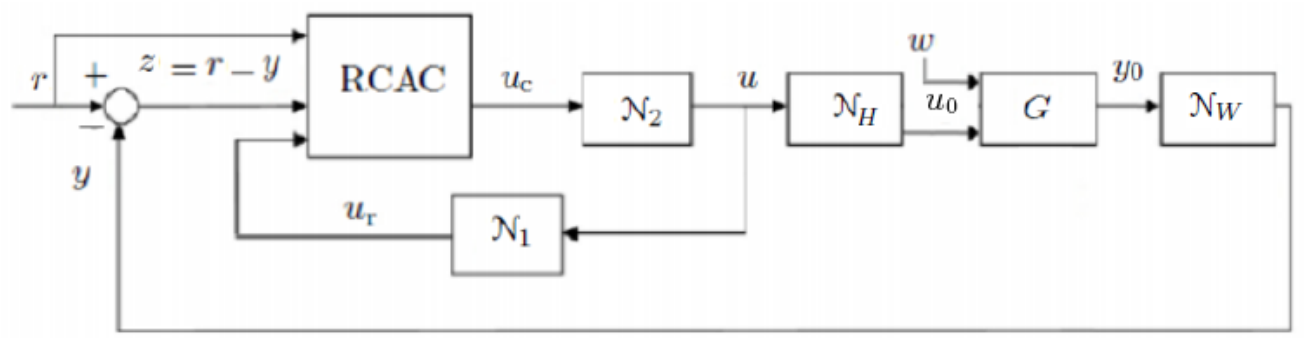

Figure 1. Hammerstein-Wiener-command-following problem with the linear system $G$, the RCAC adaptive controller, Hammerstein nonlinearity $\mathcal{N}_{H}$, Wiener nonlinearity $\mathcal{N}_{W}$, and auxiliary nonlinearities $\mathcal{N}_{1}$ and $\mathcal{N}_{2}$. The command feedforward path is optional. 


\section{Retrospective-Cost Adaptive Control}

\section{III.A. Retrospective Cost with Adaptive Regularization}

For $i \geq 1$, define the Markov parameter

$$
H_{i} \triangleq E_{1} A^{i-1} B
$$

For example, $H_{1}=E_{1} B$ and $H_{2}=E_{1} A B$. Let $\ell$ be a positive integer. Then, for all $k \geq \ell$,

$$
x(k)=A^{\ell} x(k-\ell)+\sum_{i=1}^{\ell} A^{i-1} B \mathcal{N}_{H}(u(k-i))+\sum_{i=1}^{\ell} A^{i-1} D_{1} w(k-i),
$$

and thus

$$
z(k)=\mathcal{N}_{W}\left(E_{1} A^{\ell} x(k-\ell)+\sum_{i=1}^{\ell} E_{1} A^{i-1} D_{1} w(k-i)+\bar{H} \bar{V}(k-1)+D_{2} w(k)\right)-r(k),
$$

where

$$
\bar{H} \triangleq\left[\begin{array}{ccc}
H_{1} & \cdots & H_{\ell}
\end{array}\right] \in \mathbb{R}^{1 \times \ell}
$$

and

$$
\bar{V}(k-1) \triangleq\left[\begin{array}{c}
\mathcal{N}_{H}(u(k-1)) \\
\vdots \\
\mathcal{N}_{H}(u(k-\ell))
\end{array}\right]
$$

Next, we rearrange the columns of $\bar{H}$ and the components of $\bar{V}(k-1)$ and partition the resulting matrix and vector so that

$$
\bar{H} \bar{V}(k-1)=\mathcal{H}^{\prime} V^{\prime}(k-1)+\mathcal{H} V(k-1),
$$

where $\mathcal{H}^{\prime} \in \mathbb{R}^{1 \times\left(\ell-l_{V}\right)}, \mathcal{H} \in \mathbb{R}^{1 \times l_{V}}, V^{\prime}(k-1) \in \mathbb{R}^{\ell-l_{V}}$, and $V(k-1) \in \mathbb{R}^{l_{V}}$. Then, we can rewrite (7) as

$$
z(k)=\mathcal{S}(k)+\mathcal{N}_{W}(\mathcal{H} V(k-1)),
$$

where

$$
\begin{aligned}
& \mathcal{S}(k) \triangleq \mathcal{N}_{W}\left(E_{1} A^{\ell} x(k-\ell)+\mathcal{H}^{\prime} V^{\prime}(k-1)+\sum_{i=1}^{\ell} E_{1} A^{i-1} D_{1} w(k-i)+D_{2} w(k)\right)-r(k)+\mathcal{O}(\bar{V}(k-1)), \\
& \mathcal{O}(\bar{V}(k-1)) \triangleq \mathcal{N}_{W}\left(E_{1} A^{\ell} x(k-\ell)+\sum_{i=1}^{\ell} E_{1} A^{i-1} D_{1} w(k-i)+\bar{H} \bar{V}(k-1)+D_{2} w(k)\right) \\
&-\mathcal{N}_{W}\left(E_{1} A^{\ell} x(k-\ell)+\mathcal{H}^{\prime} V^{\prime}(k-1)+\sum_{i=1}^{\ell} E_{1} A^{i-1} D_{1} w(k-i)+D_{2} w(k)\right) \\
&+\mathcal{H}^{\prime} V^{\prime}(k-1)-\mathcal{N}_{W}(\mathcal{H} V(k-1)) .
\end{aligned}
$$
the form

Next, for $j=1, \ldots, s$, we rewrite (9) with a delay of $k_{j}$ time steps, where $0 \leq k_{1} \leq k_{2} \leq \cdots \leq k_{s}$, in

$$
z\left(k-k_{j}\right)=\mathcal{S}_{j}\left(k-k_{j}\right)+\mathcal{N}_{W}\left(\mathcal{H}_{j} V_{j}\left(k-k_{j}-1\right)\right),
$$


where (10) becomes

$$
\mathcal{S}_{j}\left(k-k_{j}\right) \triangleq \mathcal{N}_{W}\left(E_{1} A^{\ell} x\left(k-k_{j}-\ell\right)+\mathcal{H}_{j}^{\prime} V_{j}^{\prime}\left(k-k_{j}-1\right)\right)-r\left(k-k_{j}\right)+\mathcal{O}\left(\bar{V}\left(k-k_{j}-1\right)\right)
$$

and (8) becomes

$$
\bar{H} \bar{V}\left(k-k_{j}-1\right)=\mathcal{H}_{j}^{\prime} V_{j}^{\prime}\left(k-k_{j}-1\right)+\mathcal{H}_{j} V_{j}\left(k-k_{j}-1\right),
$$

where $\mathcal{H}_{j}^{\prime} \in \mathbb{R}^{1 \times\left(\ell-l_{V_{j}}\right)}, \mathcal{H}_{j} \in \mathbb{R}^{1 \times l_{V_{j}}}, U_{j}^{\prime}\left(k-k_{j}-1\right) \in \mathbb{R}^{\ell-l_{V_{j}}}$, and $V_{j}\left(k-k_{j}-1\right) \in \mathbb{R}^{l_{V_{j}}}$. Now, by stacking $z\left(k-k_{1}\right), \ldots, z\left(k-k_{s}\right)$, we define the extended performance

$$
Z(k) \triangleq\left[\begin{array}{c}
z\left(k-k_{1}\right) \\
\vdots \\
z\left(k-k_{s}\right)
\end{array}\right] \in \mathbb{R}^{s} .
$$

Therefore,

$$
Z(k) \triangleq \tilde{\mathcal{S}}(k)+\tilde{\mathcal{H}} \tilde{V}(k-1)
$$

where

$$
\tilde{\mathcal{S}}(k) \triangleq\left[\begin{array}{c}
\mathcal{S}_{1}\left(k-k_{1}\right) \\
\vdots \\
\mathcal{S}_{s}\left(k-k_{s}\right)
\end{array}\right] \in \mathbb{R}^{s}
$$

$\tilde{V}(k-1)$ has the form

$$
\tilde{V}(k-1) \triangleq\left[\begin{array}{c}
\mathcal{N}_{H}\left(u\left(k-q_{1}\right)\right) \\
\vdots \\
\mathcal{N}_{H}\left(u\left(k-q_{l_{\tilde{V}}}\right)\right)
\end{array}\right] \in \mathbb{R}^{l_{\tilde{V}}},
$$

where, for $i=1, \ldots, l_{\tilde{V}}, k_{1} \leq q_{i} \leq k_{s}+\ell$, and $\tilde{\mathcal{H}} \in \mathbb{R}^{s \times l_{\tilde{V}}}$ is constructed according to the structure of $\tilde{V}(k-1)$. The vector $\tilde{V}(k-1)$ is formed by stacking $V_{1}\left(k-k_{1}-1\right), \ldots, V_{s}\left(k-k_{s}-1\right)$ and removing copies of repeated components.

Next, for $j=1, \ldots, s$, we define the retrospective performance

$$
\hat{z}_{j}\left(k-k_{j}\right) \triangleq \mathcal{S}_{j}\left(k-k_{j}\right)+\mathcal{H}_{j} \hat{V}_{j}\left(k-k_{j}-1\right),
$$

where the past controls $V_{j}\left(k-k_{j}-1\right)$ in (11) are replaced by the retrospective controls $\hat{V}_{j}\left(k-k_{j}-1\right)$. In analogy with (12), the extended retrospective performance for (14) is defined as

$$
\hat{Z}(k) \triangleq\left[\begin{array}{c}
\hat{z}_{1}\left(k-k_{1}\right) \\
\vdots \\
\hat{z}_{s}\left(k-k_{s}\right)
\end{array}\right] \in \mathbb{R}^{s}
$$

and thus is given by

$$
\hat{Z}(k)=\tilde{\mathcal{S}}(k)+\tilde{\mathcal{H}} \hat{\tilde{V}}(k-1),
$$

where the components of $\hat{\tilde{V}}(k-1) \in \mathbb{R}_{\tilde{V}}^{l}$ are the components of $\hat{V}_{1}\left(k-k_{1}-1\right), \ldots, \hat{V}_{s}\left(k-k_{s}-1\right)$ ordered in the same way as the components of $\tilde{V}(k-1)$. Subtracting (13) from (15) yields

$$
\hat{Z}(k)=Z(k)-\tilde{\mathcal{H}} \tilde{V}(k-1)+\tilde{\mathcal{H}} \hat{\tilde{V}}(k-1) .
$$


Finally, we define the retrospective cost function

$$
J(\hat{\tilde{V}}(k-1), k) \triangleq \hat{Z}^{\mathrm{T}}(k) R(k) \hat{Z}(k),
$$

where $R(k) \in \mathbb{R}^{s \times s}$ is a positive-definite performance weighting. The goal is to determine refined controls $\hat{\tilde{V}}(k-1)$ that would have provided better performance than the controls $U(k)$ that were applied to the system. The refined control values $\hat{\tilde{V}}(k-1)$ are subsequently used to update the controller.

Next, to ensure that (17) has a global minimizer, we consider the regularized cost

$$
\bar{J}(\hat{\tilde{V}}(k-1), k) \triangleq \hat{Z}^{\mathrm{T}}(k) R(k) \hat{Z}(k)+\eta(k) \hat{\tilde{V}}^{\mathrm{T}}(k-1) \hat{\tilde{V}}(k-1),
$$

where $\eta(k) \geq 0$. Substituting (16) into (18) yields

$$
\bar{J}(\hat{\tilde{V}}(k-1), k)=\hat{\tilde{V}}(k-1)^{\mathrm{T}} \mathcal{A}(k) \hat{\tilde{V}}(k-1)+\mathcal{B}(k) \hat{\tilde{V}}(k-1)+\mathcal{C}(k),
$$

where

$$
\begin{aligned}
& \mathcal{A}(k) \triangleq \tilde{\mathcal{H}}^{\mathrm{T}} R(k) \tilde{\mathcal{H}}+\eta(k) I_{l_{\tilde{V}}}, \\
& \mathcal{B}(k) \triangleq 2 \tilde{\mathcal{H}}^{\mathrm{T}} R(k)[Z(k)-\tilde{\mathcal{H}} \tilde{V}(k-1)], \\
& \mathcal{C}(k) \triangleq Z^{\mathrm{T}}(k) R(k) Z(k)-2 Z^{\mathrm{T}}(k) R(k) \tilde{\mathcal{H}} \tilde{V}(k-1)+\tilde{V}^{\mathrm{T}}(k-1) \tilde{\mathcal{H}}^{\mathrm{T}} R(k) \tilde{\mathcal{H}} \tilde{V}(k-1) .
\end{aligned}
$$

If either $\tilde{\mathcal{H}}$ has full column rank or $\eta(k)>0$, then $\mathcal{A}(k)$ is positive definite. In this case, $\bar{J}(\hat{\tilde{V}}(k-1), k)$ has the unique global minimizer

$$
\hat{\tilde{V}}(k-1)=-\frac{1}{2} \mathcal{A}^{-1}(k) \mathcal{B}(k)
$$

\section{III.B. Controller Construction}

The control $u(k)$ is given by the strictly proper time-series controller of order $n_{\mathrm{c}}$ given by

$$
u(k)=\sum_{i=1}^{n_{\mathrm{c}}} M_{i}(k) u(k-i)+\sum_{i=1}^{n_{\mathrm{c}}} N_{i}(k) z(k-i),
$$

where, for all $i=1, \ldots, n_{\mathrm{c}}, M_{i}(k) \in \mathbb{R}$, and $N_{i}(k) \in \mathbb{R}$. The control (20) can be expressed as

$$
u(k)=\theta(k) \phi(k-1),
$$

where

$$
\theta(k) \triangleq\left[M_{1}(k) \cdots M_{n_{\mathrm{c}}}(k) \cdots N_{1}(k) \cdots N_{n_{\mathrm{c}}}(k)\right] \in \mathbb{R}^{l_{u} \times 3 n_{\mathrm{c}}}
$$

and

$$
\phi(k-1) \triangleq\left[u(k-1) \cdots u\left(k-n_{\mathrm{c}}\right) \cdots z(k-1) \cdots z\left(k-n_{\mathrm{c}}\right)\right]^{\mathrm{T}} \in \mathbb{R}^{3 n_{\mathrm{c}}} .
$$

Next, let $d$ be a positive integer such that $\tilde{V}(k-1)$ contains $u_{0}(k-d)$ and define the cumulative cost function

$$
J_{\mathrm{R}}(\theta, k) \triangleq \sum_{i=d+1}^{k} \lambda^{k-i}\left\|\phi^{\mathrm{T}}(i-d-1) \theta^{\mathrm{T}}(k)-{\hat{u_{0}}}^{\mathrm{T}}(i-d)\right\|^{2}+\lambda^{k}\left(\theta(k)-\theta_{0}\right) P_{0}^{-1}\left(\theta(k)-\theta_{0}\right)^{\mathrm{T}},
$$

where $\|\cdot\|$ is the Euclidean norm, and $\lambda \in(0,1]$ is the forgetting factor. Minimizing (21) yields

$$
\begin{aligned}
\theta^{\mathrm{T}}(k)= & \theta^{\mathrm{T}}(k-1)+\beta(k) P(k-1) \phi(k-d-1) \cdot\left[\phi^{\mathrm{T}}(k-d) P(k-1) \phi(k-d-1)+\lambda(k)\right]^{-1} \\
& \cdot\left[\phi^{\mathrm{T}}(k-d-1) \theta^{\mathrm{T}}(k-1)-\hat{u}_{0}^{\mathrm{T}}(k-d)\right],
\end{aligned}
$$


where $\beta(k)$ is either zero or one. The error covariance is updated by

$$
\begin{aligned}
P(k)= & \beta(k) \lambda^{-1} P(k-1)+[1-\beta(k)] P(k-1)-\beta(k) \lambda^{-1} P(k-1) \phi(k-d-1) \\
& \cdot\left[\phi^{\mathrm{T}}(k-d-1) P(k-1) \phi(k-d)+\lambda\right]^{-1} \cdot \phi^{\mathrm{T}}(k-d-1) P(k-1) .
\end{aligned}
$$

We initialize the error covariance matrix as $P(0)=\alpha I_{3 n_{\mathrm{c}}}$, where $\alpha>0$. Note that when $\beta(k)=0, \theta(k)=$ $\theta(k-1)$ and $P(k)=P(k-1)$. Therefore, setting $\beta(k)=0$ switches off the controller adaptation, and thus freezes the control gains. When $\beta(k)=1$, the controller is allowed to adapt.

\section{III.C. Auxiliary Nonlinearities and the Adaptive RCAC Controller}

To account for the presence of the nonlinearities $\mathcal{N}_{H}$ and $\mathcal{N}_{W}$, the RCAC controller uses two auxiliary nonlinearities. The auxiliary nonlinearity $\mathcal{N}_{2}$ modifies the RCAC controller output $u_{\mathrm{c}}$ to produce the Hammerstein-Wiener plant input $u$, while the auxiliary nonlinearity $\mathcal{N}_{1}$ modifies $u$ to obtain the regressor input $u_{\mathrm{r}}$. The auxiliary nonlinearities $\mathcal{N}_{1}$ and $\mathcal{N}_{2}$ are chosen based on limited knowledge of the nonlinearities $\mathcal{N}_{H}$ and $\mathcal{N}_{W}$. For details see. ${ }^{13}$

\section{III.C.1. Auxiliary Nonlinearity $\mathcal{N}_{1}$}

Define the saturation function $\operatorname{sat}_{a}$ by

$$
\mathcal{N}_{1}\left(u_{\mathrm{c}}\right)=\operatorname{sat}_{a}\left(u_{\mathrm{c}}\right)= \begin{cases}-a, & \text { if } \quad u_{\mathrm{c}}<-a, \\ u_{\mathrm{c}}, & \text { if }-a \leq u_{\mathrm{c}} \leq a, \\ a, & \text { if } u_{\mathrm{c}}>a,\end{cases}
$$

where $a>0$ is the saturation level. For minimum-phase plants, the auxiliary nonlinearity $\mathcal{N}_{1}$ is not needed, and thus the saturation level $a$ is chosen to be a large number. For nonminimum-phase plants, the saturation level $a$ is chosen to tune the transient behavior. In addition to the transient behavior, the saturation level is chosen based on the magnitude of the control input needed to follow the command $r$. This level depends on the range of the input nonlinearity $\mathcal{N}_{H}$ as well as the gain of the linear system $G$ at frequencies in the spectrum of $r$.

\section{III.C.2. Auxiliary Nonlinearity $\mathcal{N}_{2}$}

To construct $\mathcal{N}_{2}$, we assume that the intervals of monotonicity of the input nonlinearity $\mathcal{N}_{H}$ are known; no further modeling information about $\mathcal{N}_{H}$ is needed. Let $I_{1}, I_{2}, \ldots$ be intervals that partition the real numbers. If $\mathcal{N}_{H}$ is nondecreasing on $I_{i}$, then $\mathcal{N}_{2}\left(u_{\mathrm{r}}\right)=u_{\mathrm{r}}$ for all $u_{\mathrm{r}} \in I_{i}$. Alternatively, if $\mathcal{N}_{H}$ is nonincreasing on $I_{i}=\left(p_{i}, q_{i}\right)$, then $\mathcal{N}_{2}\left(u_{\mathrm{r}}\right)=p_{i}+q_{i}-u_{\mathrm{r}} \in I_{i}$ for all $u_{\mathrm{r}} \in I_{i}$. Finally, if $\mathcal{N}_{H}$ is constant on $I_{i}$, then either choice can be used. Let $\mathcal{R}_{a}(f)$ denote the range of the function $f$ with arguments in [-a,a]. hold:

Proposition III.1. Assume that $\mathcal{N}_{2}$ is constructed by the above rule. Then the following statements

i) $\mathcal{N}_{H} \circ \mathcal{N}_{2}$ is piecewise nondecreasing.

ii) $\mathcal{R}_{a}\left(\mathcal{N}_{H} \circ \mathcal{N}_{2}\right)=\mathcal{R}_{a}\left(\mathcal{N}_{H}\right)$.

Proof III.1. Let $I_{i}=\left(p_{i}, q_{i}\right)$. We first assume that $\mathcal{N}_{H}$ is nondecreasing on $I_{i}$. Since $\mathcal{N}_{2}\left(u_{\mathrm{r}}\right)=u_{\mathrm{r}}$ for all $u_{\mathrm{r}} \in I_{i}$, it follows that $\mathcal{N}_{H} \circ \mathcal{N}_{2}\left(u_{\mathrm{r}}\right)=\mathcal{N}_{H}\left(u_{\mathrm{r}}\right)$ for all $u_{\mathrm{r}} \in I_{i}$. Hence $\mathcal{N}_{H} \circ \mathcal{N}_{2}$ is nondecreasing on $I_{i}$. 
Next, assume that $\mathcal{N}_{H}$ is nonincreasing on $I_{i}$. Let $u_{\mathrm{r}, 1}, u_{\mathrm{r}, 2} \in I_{i}$, where $u_{\mathrm{r}, 1} \leq u_{\mathrm{r}, 2}$. Since $\mathcal{N}_{2}\left(u_{\mathrm{r}}\right)=p_{i}+q_{i}-u_{\mathrm{r}}$ for all $u_{\mathrm{r}} \in I_{i}$, it follows that

$$
u_{2} \triangleq p_{i}+q_{i}-u_{\mathrm{r}, 2} \leq u_{1} \triangleq p_{i}+q_{i}-u_{\mathrm{r}, 1} .
$$

Therefore, since $\mathcal{N}_{H}$ is nonincreasing on $I_{i}$, it follows that $\mathcal{N}_{H}\left(\mathcal{N}_{2}\left(u_{\mathrm{r}, 1}\right)\right)=\mathcal{N}_{H}\left(u_{1}\right) \leq \mathcal{N}_{H}\left(u_{2}\right)=\mathcal{N}_{H}\left(\mathcal{N}_{2}\left(u_{\mathrm{r}, 2}\right)\right)$. Thus, $\mathcal{N}_{H} \circ \mathcal{N}_{2}$ is nondecreasing on $I_{i}$.

Finally, to prove ii), assume that $\mathcal{N}_{H}$ is nondecreasing on $I_{i}$. Since $\mathcal{N}_{2}\left(u_{\mathrm{r}}\right)=u_{\mathrm{r}}$ for all $u_{\mathrm{r}} \in I_{i}$, it follows that $\mathcal{N}_{2}\left(I_{i}\right)=I_{i}$, that is, $\mathcal{N}_{2}: I_{i} \rightarrow I_{i}$ is onto. Alternatively, assume that $\mathcal{N}_{H}$ is nonincreasing on $I_{i}$. Then $\mathcal{N}_{2}\left(u_{\mathrm{r}}\right)=p_{i}+q_{i}-u_{\mathrm{r}} \in I_{i}$. Let $y=\mathcal{N}_{2}\left(u_{\mathrm{r}}\right)$ be an element of the codomain, consider $y=p_{i}+q_{i}-u_{\mathrm{r}}$, and solve for $u_{\mathrm{r}}$ yields $u_{\mathrm{r}}=p_{i}+q_{i}-y$. Thus, for all $y \in I_{i}$, there exists $p_{i}+q_{i}-y \in I_{i}$ such that $\mathcal{N}_{2}\left(p_{i}+q_{i}-y\right)=y$. Therefore, $\mathcal{N}_{2}: I_{i} \rightarrow I_{i}$ is onto. Hence, $\mathcal{R}_{a}\left(\mathcal{N}_{H} \circ \mathcal{N}_{2}\right)=\mathcal{R}_{a}\left(\mathcal{N}_{H}\right)$.

Example III.1. Consider the input nonlinearity $\mathcal{N}_{H}(u)=-\operatorname{sat}(\Psi[u])$ with $u(t)=2 \sin (2 \pi t)$. For each interval of a partition $0=t_{0}<t_{1}<\cdots<t_{l}=10$, the output of the play operator for $t \in\left[t_{j-1}, t_{j}\right]$ expressed as

$$
\Psi[u](t)=\max \left\{u(t)-0.5, \min \left\{u(t)+0.5, \Psi[u]\left(t_{j-1}\right)\right\}\right\} .
$$

The input nonlinearity $\mathcal{N}_{H}(u)=-\operatorname{sat}(\Psi[u])$ is nonincreasing for all $u \in \mathcal{R}_{a}$ as shown in Figure 2(a). Let $\mathcal{N}_{2}\left(u_{\mathrm{r}}\right)=-u_{\mathrm{r}}$ according to Proposition III.1. Figure 2(c) shows that the composite nonlinearity $\mathcal{N}_{H} \circ \mathcal{N}_{2}$ is nondecreasing. Note that $\mathcal{R}_{2}\left(\mathcal{N}_{H} \circ \mathcal{N}_{2}\right)=\mathcal{R}_{2}\left(\mathcal{N}_{H}\right)$.

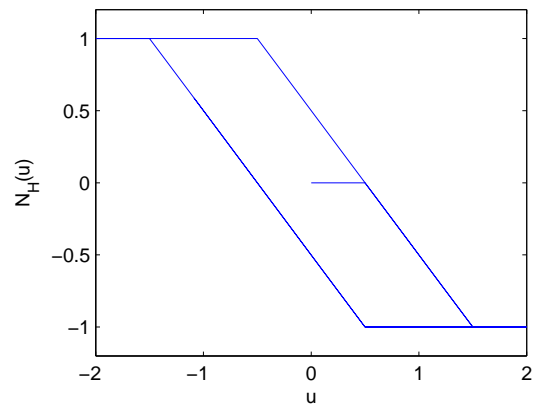

(a)

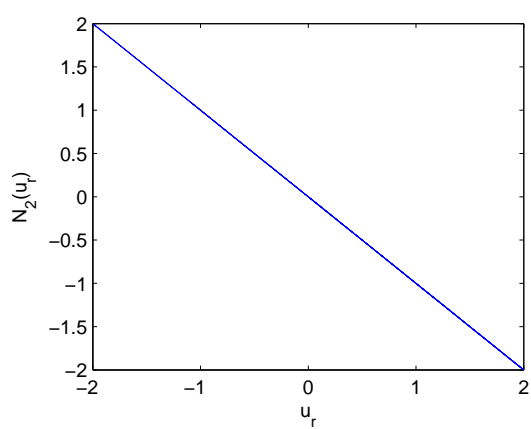

(b)

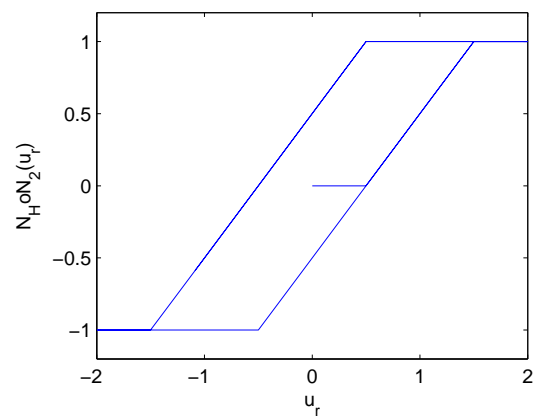

(c)

Figure 2. Example III.1 (a) Input nonlinearity $\mathcal{N}_{H}(u)=-\operatorname{sat}(\Psi[u])$, (b) Auxiliary nonlinearity $\mathcal{N}_{2}\left(u_{\mathrm{r}}\right)$ given by (24), and (c) The composite nonlinearity $\mathcal{N}_{H} \circ \mathcal{N}_{2}$. Note that $\mathcal{N}_{H} \circ \mathcal{N}_{2}$ is piecewise nondecreasing and $\mathcal{R}_{2}\left(\mathcal{N}_{H} \circ \mathcal{N}_{2}\right)=\mathcal{R}_{2}\left(\mathcal{N}_{H}\right)$.

Example III.2. Consider $u(t)=2 \sin (2 \pi t)$ and input nonlinearity shown in Figure $3(\mathrm{a})$, which is given by

$$
\mathcal{N}_{\mathcal{H}}(u)= \begin{cases}-\operatorname{sat}_{0.5}(\Psi[u]), & \text { if }-2 \leq u \leq 1 \\ \frac{u^{2}}{5}-0.7, & \text { if } 1<u \leq 2\end{cases}
$$


We use $\Psi[u]$ considered in Example (III.1). Let

$$
\mathcal{N}_{2}\left(u_{\mathrm{r}}\right)= \begin{cases}-u_{\mathrm{r}}, & \text { if }-2 \leq u \leq 1, \\ u_{\mathrm{r}}, & \text { if } 1<u \leq 2,\end{cases}
$$

according to Proposition 3.1. Figure 3(c) shows that the composite nonlinearity $\mathcal{N}_{H} \circ \mathcal{N}_{2}$ is piecewise nondecreasing. Note that $\mathcal{R}_{2}\left(\mathcal{N}_{H} \circ \mathcal{N}_{2}\right)=\mathcal{R}_{2}\left(\mathcal{N}_{H}\right)$.

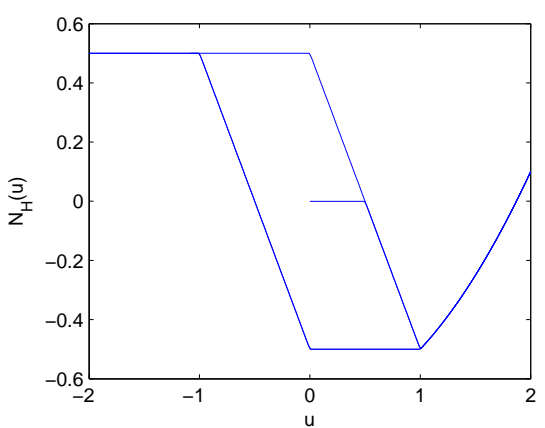

(a)

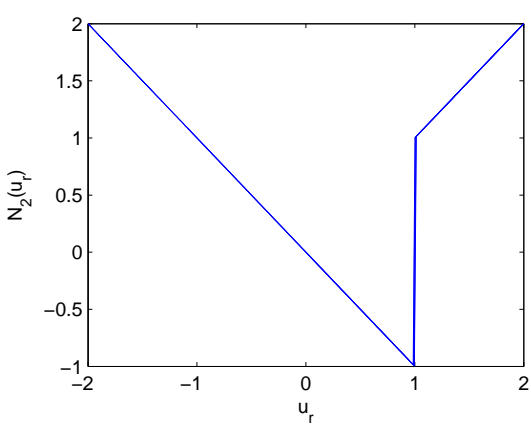

(b)

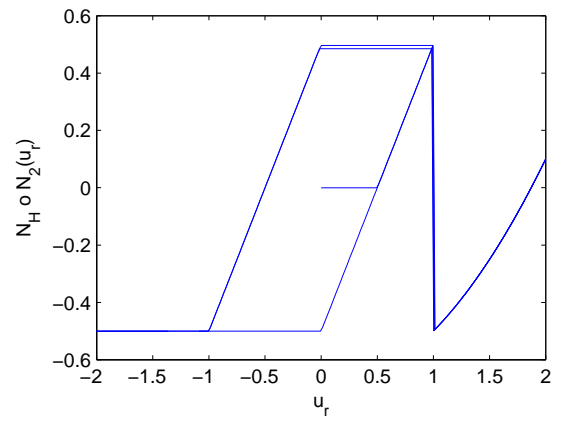

(c)

Figure 3. Example III.2 (a) Input nonlinearity $\mathcal{N}_{H}(u)(23)$, (b) Auxiliary nonlinearity $\mathcal{N}_{2}\left(u_{\mathrm{r}}\right)$ given by (24), and, (c) The composite nonlinearity $\mathcal{N}_{H} \circ \mathcal{N}_{2}$. Note that $\mathcal{N}_{H} \circ \mathcal{N}_{2}$ is piecewise nondecreasing and $\mathcal{R}_{2}\left(\mathcal{N}_{H} \circ \mathcal{N}_{2}\right)=\mathcal{R}_{2}\left(\mathcal{N}_{H}\right)$.

Knowledge of only the intervals of monotonicity of $\mathcal{N}_{H}$ is needed to modify the controller output $u_{\mathrm{r}}$ such that the composite nonlinearity $\mathcal{N}_{H} \circ \mathcal{N}_{2}$ is piecewise nondecreasing. It thus follows that the known Markov parameters $\tilde{H}$ of $G$ capture correct sign information of the linearized Markov parameters for the Hammerstein system since $\mathcal{N}_{H} \circ \mathcal{N}_{2}$ is piecewise nondecreasing. For details, see. ${ }^{14}$

\section{NUMERICAL EXAMPLES}

We illustrate RCAC for SISO command-following problems with a linear plant in the presence of Hammerstein-Wiener nonlinearities. In all examples, we assume that at least one of the nonzero Markov parameters of $G$ is known. For convenience, each example is constructed such that the first nonzero Markov parameter $H_{d}=1$, where $d$ is the relative degree of $G$. RCAC generates a control signal $u_{\mathrm{c}}$ that attempts to minimize a performance matrix based on the performance variable $z$ in the presence of the command signal $r$, the input nonlinearity $\mathcal{N}_{H}$, and the output nonlinearity $\mathcal{N}_{W}$. We assume that measurements of $z$ are available for feedback; however, measurements of $u_{0}$ and $y_{0}$ are not available. In all cases, we initialize the adaptive controller to be zero, that is, $\theta(0)=0$. We do not use a forgetting factor in any of the examples of this paper, that is, $\lambda=1$ for all examples. 
To illustrate the effect of nonlinearities on the closed-loop performance, we first remove the HammersteinWiener nonlinearities and simulate the open-loop system for the first 100 time steps. Then, at $k=100$, we turn the adaptation on, and let RCAC adapt to the linear system for 600 time steps. We use a solid trace to show the simulation results for the closed-loop system before inserting the Hammerstein-Wiener nonlinearities. Next, at $k=600$, we introduce the Hammerstein-Wiener nonlinearities and let RCAC adapt in the presence of the Hammerstein-Wiener nonlinearities. We use dotted markers for $k \geq 600$ to illustrate performance in the presence of the input and output nonlinearities, $\mathcal{N}_{H}(u(k))$ and $\mathcal{N}_{W}\left(y_{0}(k)\right)$, when $u(k)$ and $y(k)$ are obtained from the closed-loop system with RCAC.

\section{Numerical Examples: Memoryless-Memoryless Nonlinearities}

Example V.1. We consider the minimum-phase asymptotically stable linear plant

$$
G(z)=\frac{z-0.9}{(z-0.8)(z-0.7)}
$$

with the deadband input nonlinearity

$$
\mathcal{N}_{H}(u)= \begin{cases}u, & \text { if } u<-0.3, \\ 0, & \text { if }-0.3 \leq u \leq 0.3, \\ u, & \text { if } u>0.3\end{cases}
$$

and the cubic output nonlinearity

$$
\mathcal{N}_{W}\left(y_{0}\right)=y_{0}^{3} .
$$

Since these nonlinearities are nondecreasing but otherwise assumed to be unknown we choose $\mathcal{N}_{1}(u)=u$ and $\mathcal{N}_{2}\left(u_{\mathrm{c}}\right)=\operatorname{sat}_{a}\left(u_{\mathrm{c}}\right)$. We let $a=2, n_{\mathrm{c}}=15$, and $P_{0}=0.2 I_{3 n_{\mathrm{c}}}$. We consider the command signal $r(k)=\sin (0.25 \pi k)$ and the disturbance signal $w(k)=0.5 \sin (0.5 \pi k)$. Figure 4 shows the simulation results.

Example V.2. We consider the nonminimum-phase asymptotically stable linear plant

$$
G(z)=\frac{z-1.1}{(z-0.9)(z+0.9)}
$$

with the sigmoidal input nonlinearity

$$
\mathcal{N}_{H}(u)=\tanh (u)
$$

and the deadband output nonlinearity

$$
\mathcal{N}_{W}\left(y_{0}\right)= \begin{cases}y_{0}, & \text { if } y_{0}<-0.3 \\ 0, & \text { if }-0.3 \leq y_{0} \leq 0.3 \\ y_{0}, & \text { if } y_{0}>0.3\end{cases}
$$

Since these nonlinearities are nondecreasing, we choose $\mathcal{N}_{1}(u)=u$ and $\mathcal{N}_{2}\left(u_{\mathrm{c}}\right)=\operatorname{sat}_{a}\left(u_{\mathrm{c}}\right)$. The command signal $r(k)=0.8 \sin (0.25 \pi k)+1.6 \sin (\pi k)$ and the disturbance signal $w(k)=0.5 \sin (0.25 \pi k)$ are considered. We let $a=7, n_{\mathrm{c}}=20$, and $P_{0}=0.7 I_{3 n_{\mathrm{c}}}$. Figure 5 shows the simulation results.

Example V.3. We consider the nonminimum-phase asymptotically stable linear plant

$$
G(z)=\frac{1}{z-1},
$$




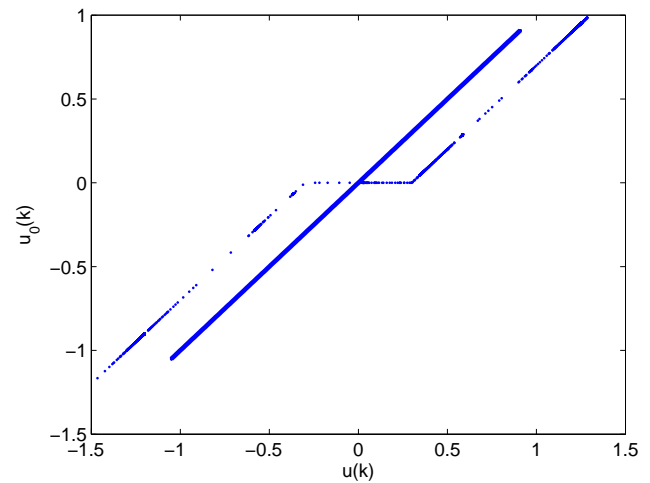

(a)

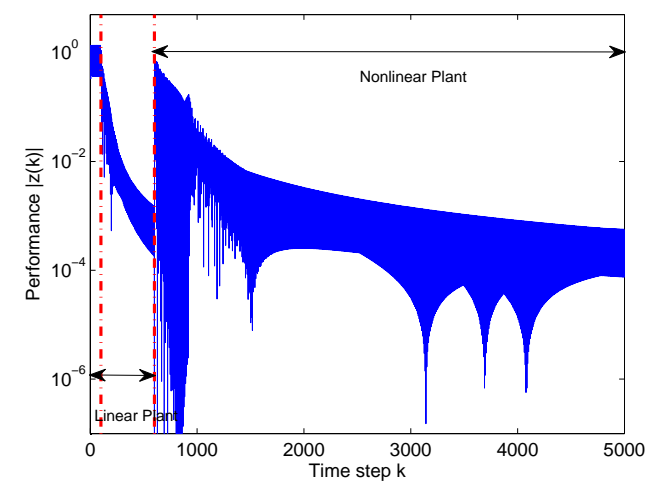

(c)

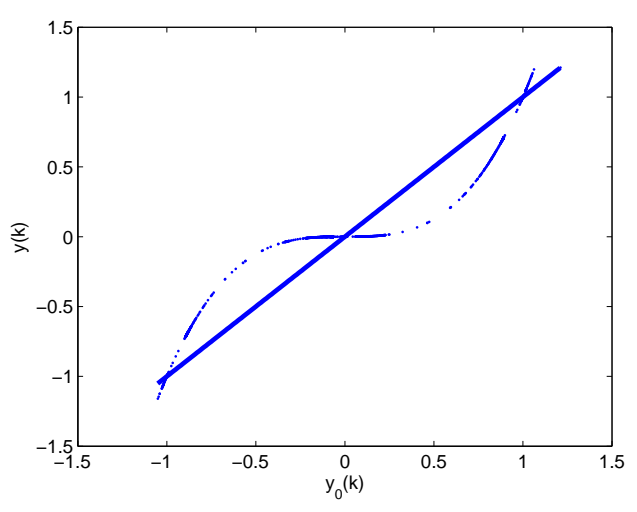

(b)

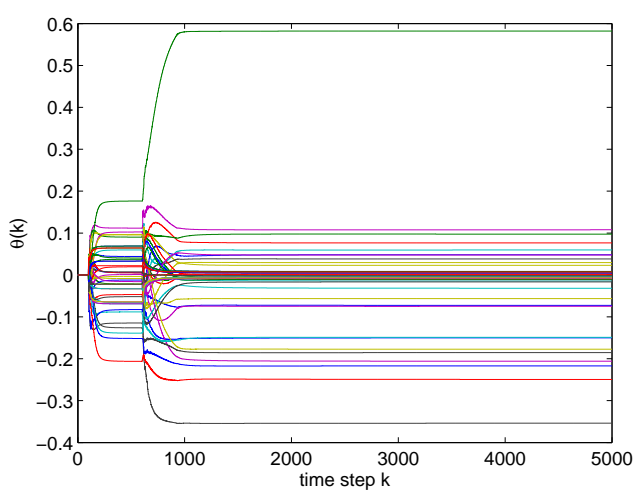

(d)

Figure 4. Example V.1. (a) shows the memoryless input nonlinearity $\mathcal{N}_{H}$ given by (26). (b) shows the memoryless output nonlinearity $\mathcal{N}_{W}$ given by (27). (c) shows the closed-loop response to the command signal $r(k)=\sin (0.5 \pi k)$ and the disturbance signal $w(k)=0.5 \sin (0.5 \pi k)$ with the linear plant (25) and the nonlinearities of (a) and (b). (d) shows the evolution of the controller gain vector $\theta$.

with the deadband input nonlinearity

$$
\mathcal{N}_{H}(u)= \begin{cases}u, & \text { if } u<-0.5 \\ 0, & \text { if }-0.5 \leq u \leq 0.5 \\ u, & \text { if } u>0.5\end{cases}
$$

and the sigmoidal output nonlinearity

$$
\mathcal{N}_{W}\left(y_{0}\right)=\tanh \left(y_{0}\right)
$$

The command signal $r(k)=1 \sin \left(\frac{\pi}{6} k\right)$ and the disturbance signal $w(k)=0.5 \sin (0.5 \pi k)$ are considered. Since this nonlinearities are nondecreasing, we choose $\mathcal{N}_{1}(u)=u$ and $\mathcal{N}_{2}\left(u_{\mathrm{c}}\right)=\operatorname{sat}_{a}\left(u_{\mathrm{c}}\right)$. We let $a=7, n_{\mathrm{c}}=7$, and $P_{0}=0.8 I_{3 n_{\mathrm{c}}}$. Figure 6 shows the simulation results.

Example V.4. We consider the asymptotically stable, minimum-phase plant

$$
G(z)=\frac{(z-0.5)(z-0.9)}{(z-0.7)(z-0.5-\jmath 0.5)(z-0.5+\jmath 0.5)},
$$

with the input nonlinearity

$$
\mathcal{N}(u)=\cos (2 u)
$$




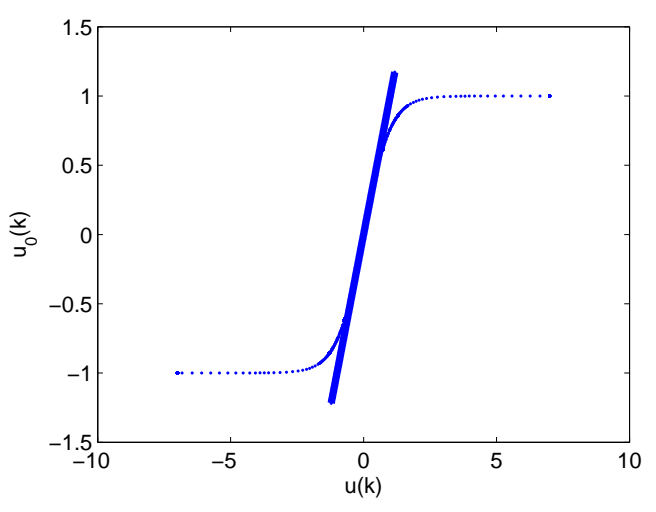

(a)

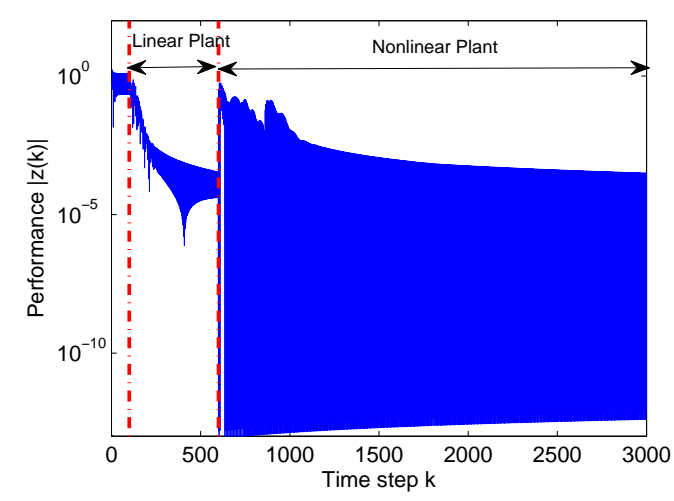

(c)

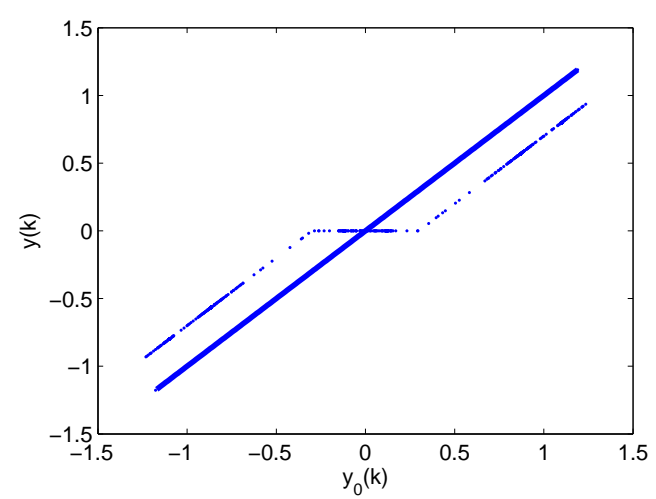

(b)

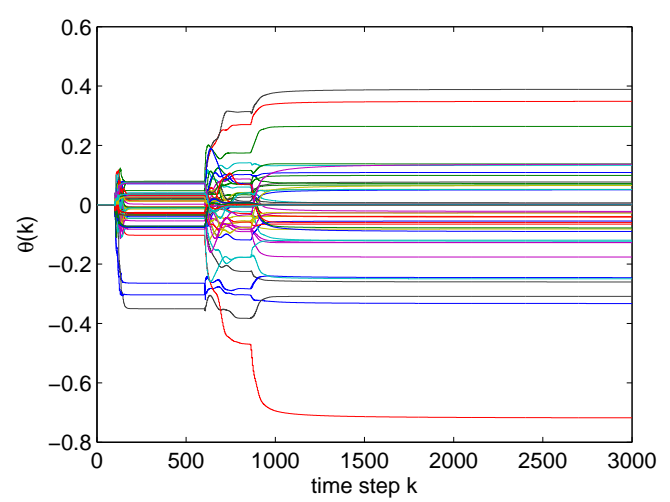

(d)

Figure 5. Example V.2. (a) shows the memoryless input nonlinearity $\mathcal{N}_{H}$ given by (33). (b) shows the deadband output nonlinearity $\mathcal{N}_{W}$ given by (32). (c) shows the closed-loop response to the command signal $r(k)=0.8 \sin (0.25 \pi k)+1.6 \sin (\pi k)$ and the disturbance signal $w(k)=0.5 \sin (0.25 \pi k)$ with the linear plant (25) and the nonlinearities of (a) and (b). (d) shows the evolution of the controller gain vector $\theta$.

and the sigmoidal output nonlinearity

$$
\mathcal{N}_{W}\left(y_{0}\right)=\tanh \left(y_{0}\right)
$$

The input $\mathcal{N}_{H}$ is neither one-to-one nor onto and satisfies $\mathcal{N}_{H}(0)=1$. As shown in Figure $7($ a $), \mathcal{N}_{H}(u)$ is increasing for all $u \in\left(\left(n-\frac{1}{2}\right) \pi, n \pi\right), n \in \mathbb{Z}$, and decreasing for all $u \in\left(n \pi,\left(n+\frac{1}{2}\right) \pi\right), n \in \mathbb{Z}$. We consider the single-tone sinusoidal command $r(k)=1 \sin (0.2 \pi k)$ and the disturbance signal $w(k)=0.25 \sin (0.5 \pi k)$. We let $\mathcal{N}_{1}\left(u_{\mathrm{c}}\right)=\operatorname{sat}_{a}\left(u_{\mathrm{c}}\right)$, where $a=10^{6}, n_{\mathrm{c}}=14, P_{0}=0.1 I_{3 n_{\mathrm{c}}}, \eta_{0}=0$, and $\mathcal{H}=H_{1}$. We choose $\mathcal{N}_{2}\left(u_{\mathrm{r}}\right)=u_{\mathrm{r}}$ for the intervals where $\mathcal{N}_{H}$ is increasing, and $\mathcal{N}_{2}\left(u_{\mathrm{r}}\right)=-u_{\mathrm{r}}+(2 n+1 / 2) \pi$ for the intervals where $\mathcal{N}_{H}$ is decreasing. We turn on the RCAC controller at $k=100$ for the Hammerstein-Wiener system. Figure 7 shows the simulation results.

\section{Numerical Examples: Hysteretic-Hysteretic Nonlinearities}

\section{VI.A. The Prandtl-Ishlinskii Model}

Smart actuators such as piezoelectric and magnetostrictive actuators have shown rate-dependent hysteresis nonlinearity, where the hysteresis nonlinearity increases as the rate of the input increases. ${ }^{17,19}$ In this paper, we use the rate-dependent Prandtl-Ishlinskii model to represent rate-dependent hysteresis nonlinear- 


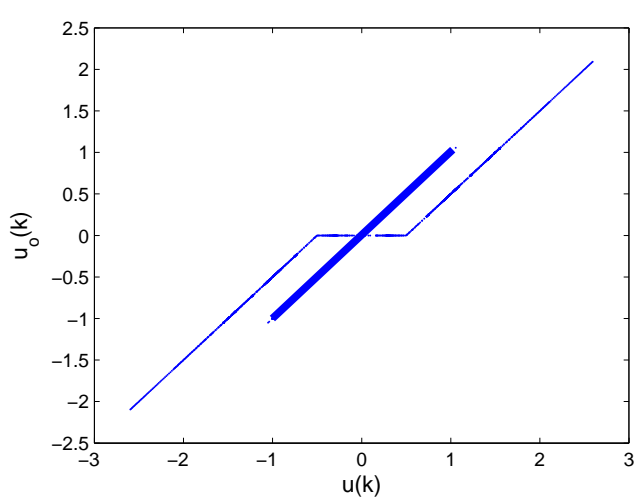

(a)

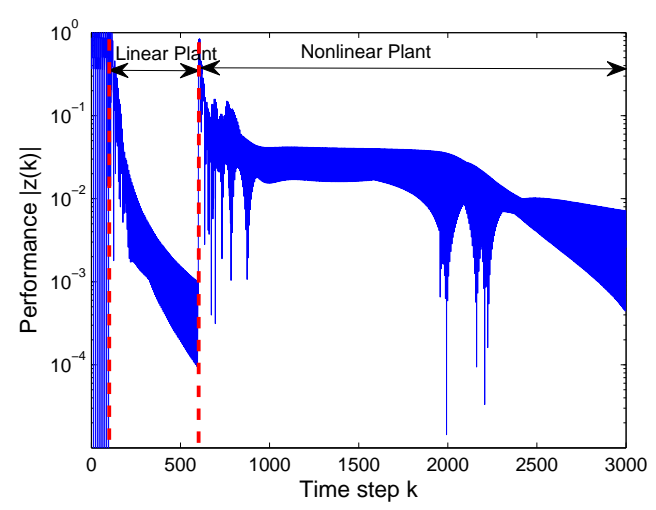

(c)

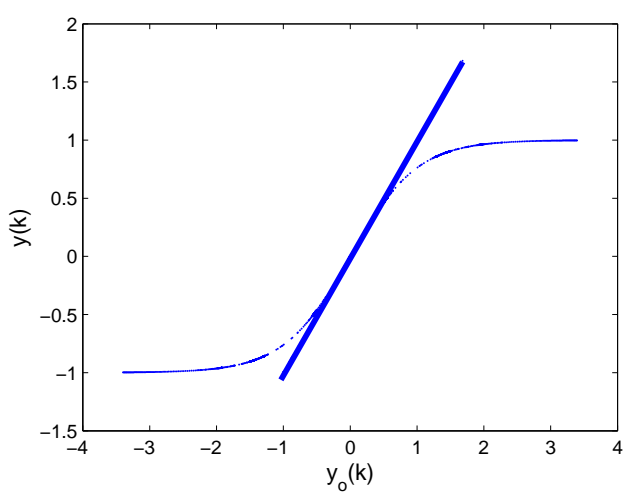

(b)

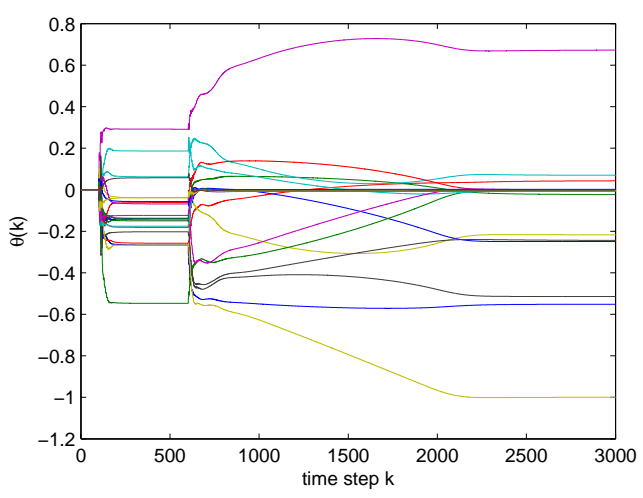

(d)

Figure 6. Example V.3. (a) shows the deadband input nonlinearity $\mathcal{N}_{W}$ given by (32). (b) shows the sigmoidal output nonlinearity $\mathcal{N}_{H}$ given by (33). (c) shows the closed-loop response to the command signal $r(k)=1 \sin \left(\frac{\pi}{6} k\right)$ and the disturbance signal $w(k)=0.5 \sin (0.5 \pi k)$ with the linear plant (31) and the nonlinearities of (a) and (b). (d) shows the evolution of the controller gain vector $\theta$.

ities. This model can characterize rate-dependent hysteresis nonlinearity in piezoelectric actuators. ${ }^{17}$ This model is used in this paper to represent the hysteretic input and the output nonlinearities.

The space of absolutely continuous functions is denoted by $A C(0, T)$. Let the input signal $v(t) \in$ $A C(0, T)$, and let $\rho_{i}(\dot{v}(t)) \in A C(0, T)$ for all $i \in\{0,1, \ldots, n\}$, where $n \in \mathbb{N}$. Then $\rho_{i}(\dot{v}(t))$ is chosen such that

$$
0=\rho_{0}(\dot{v}(t)) \leq \rho_{1}(\dot{v}(t)) \leq \rho_{2}(\dot{v}(t)) \leq \cdots \leq \rho_{n}(\dot{v}(t)) .
$$

The output $g_{i}(t)$ of the rate-dependent play operator is denoted as

$$
g_{i}(t)=\Psi_{\rho_{i}(\dot{v}(t))}\left[v, x_{i}\right](t)
$$

for inputs and thresholds that are piecewise linear, that is, linear in each interval of a partition $0=t_{0}<$ $t_{1}<\cdots<t_{l}=T$. The output of the rate-dependent play operator for $t \in\left(t_{j-1}, t_{j}\right]$ can be expressed as

$$
g_{i}(t)=\max \left\{v(t)-\rho_{i}(\dot{v}(t)), \min \left\{v(t)+\rho_{i}(\dot{v}(t)), g_{i}\left(t_{j-1}\right)\right\}\right\}
$$

with the initial condition

$$
g_{i}(0)=\max \left\{v(0)-\rho_{i}(\dot{v}(0)), \min \left\{v(0)+\rho_{i}(\dot{v}(0)), x_{i}\right\}\right\} .
$$

The rate-dependent Prandtl-Ishlinskii model is constructed as a superposition of rate-dependent play operators. ${ }^{18}$ The output of this model can be expressed as

$$
\Phi[v](t):=a_{0} v(t)+\sum_{i=1}^{n} a_{i} \Psi_{\rho_{i}(\dot{v}(t))}\left[v, x_{i}\right](t),
$$




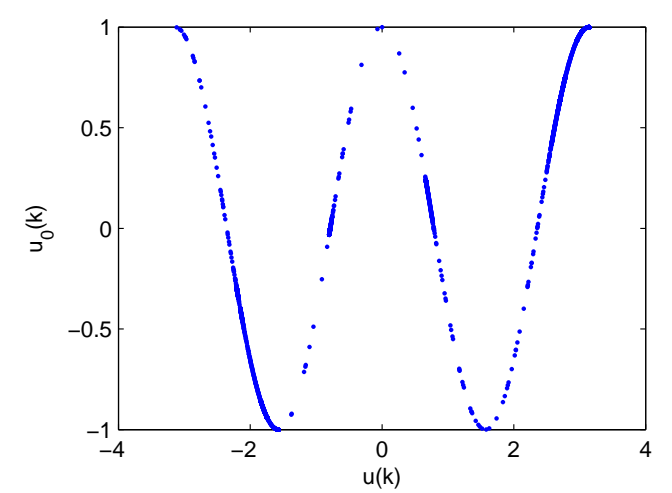

(a)

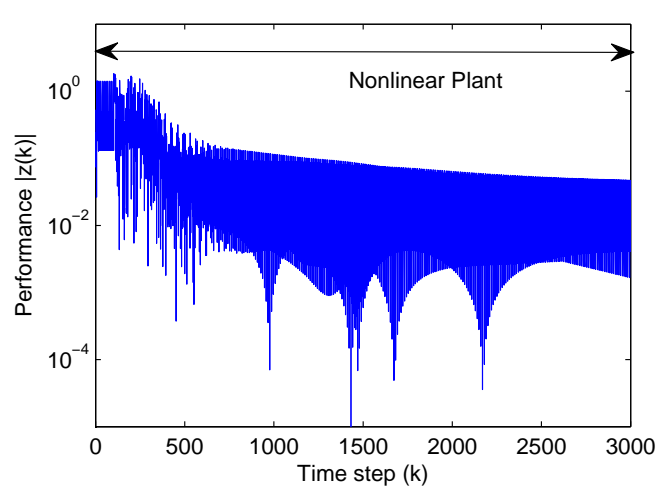

(c)

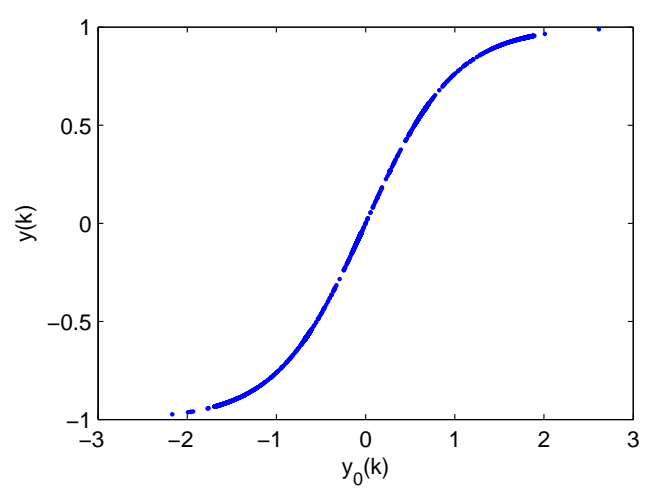

(b)

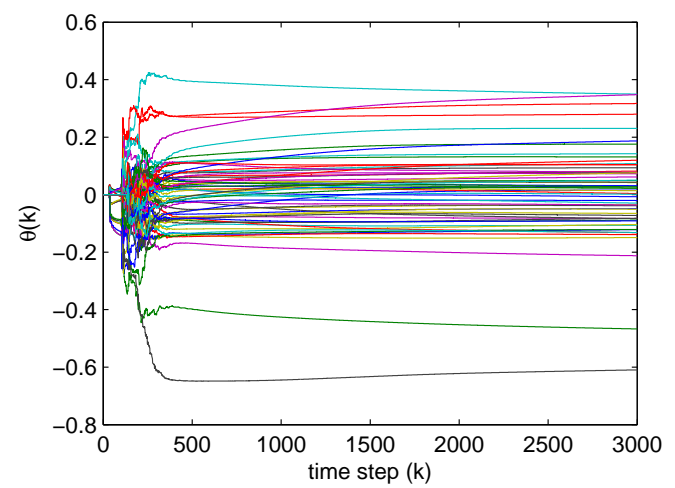

(d)

Figure 7. Example V.4. (a) shows the memoryless input nonlinearity $\mathcal{N}_{H}$ given by (35). (b) shows the sigmoidal output nonlinearity nonlinearity $\mathcal{N}_{W}$ given by (36). (c) shows the closed-loop response to the command $\operatorname{signal} r(k)=1 \sin (0.25 \pi k)$ and the disturbance signal $w(k)=0.25 \sin (0.5 \pi k)$ with the linear plant (34) and the nonlinearities of (a) and (b). (d) shows the evolution of the controller gain vector $\theta$.

where $a_{0}, \cdots, a_{n}$ are positive constants.

Next, we define the rate-dependent threshold function

$$
\rho_{i}(\dot{v}(t)):=\zeta_{i}+\eta_{i}|\dot{v}(t)|
$$

where $\zeta_{i}$ and $\eta_{i}$ are positive constants. The rate-dependent Prandtl-Ishlinskii model specializes to the rateindependent Prandtl-Ishlinskii model when $\dot{v}(t)=0$. The rate-dependent model

$$
\Phi_{\Gamma}[v](t)=(\Phi \circ \Gamma)[v](t)
$$

generalizes the rate-dependent Prandtl-Ishlinskii model (40), where $\Gamma$ is a memoryless, continuous, strictly monotonic function.

Example VI.1. We consider the linear plant

$$
G(z)=\frac{1}{z+1}
$$

with hysteretic input nonlinearity

$$
v(k)=\mathcal{N}_{H}(u(k))=\Phi[u](k)
$$

composed of five play operators $n=5$ and thresholds

$$
\rho_{i}(\dot{u}(t))=\zeta_{i}+\eta_{i}|\dot{u}(t)|,
$$


where $\zeta_{1}=0.0315, \zeta_{2}=0.0756, \zeta_{3}=0.1134, \zeta_{4}=0.1512, \zeta_{5}=0.189, \eta_{1}=0.2, \eta_{2}=0.4, \eta_{3}=0.6, \eta_{4}=0.8$, and $\eta_{5}=1.0$. The weights $a_{1}=0.18, a_{2}=0.162, a_{3}=0.15, a_{4}=0.138$, and $a_{5}=0.12$ and the initial conditions $x_{i}=0$ are considered. We consider hysteretic output nonlinearity

$$
y(k)=\mathcal{N}_{W}\left(y_{0}(k)\right)=\Phi\left[y_{0}\right](k)
$$

composed of three play operators $n=3$ and thresholds

$$
\rho_{i}\left(\dot{y}_{0}(t)\right)=\zeta_{i}+\eta_{i}\left|\dot{y}_{0}(t)\right|
$$

where $\zeta_{1}=0.2, \zeta_{2}=0.2, \zeta_{3}=0.2, \eta_{1}=0.4, \eta_{2}=0.2, \eta_{3}=0.3$. The weights $a_{0}=0.6, a_{1}=0.4, a_{2}=0.3$, and $a_{3}=0.2$ and the initial conditions $x_{i}=0$ are considered. We let $a=5, n_{\mathrm{c}}=30$, and $P_{0}=2 I_{3 n_{\mathrm{c}}}$. We use the command signal $\sin (0.25 \pi k)+0.5 \sin (0.5 \pi k)$ and the disturbance signal $w(k)=0.5 \sin (0.5 \pi k)$. Figure 8

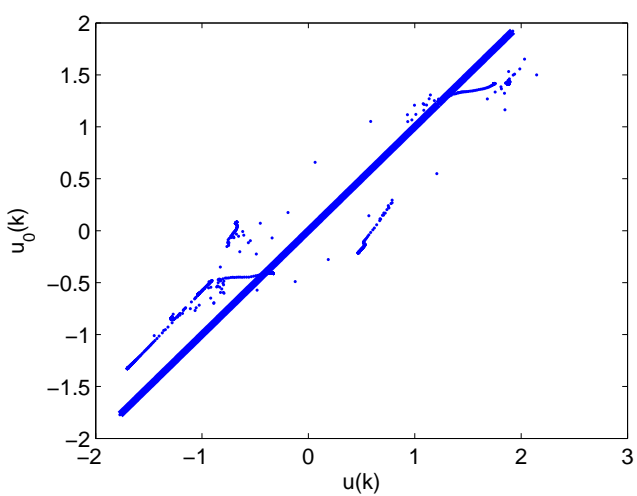

(a)

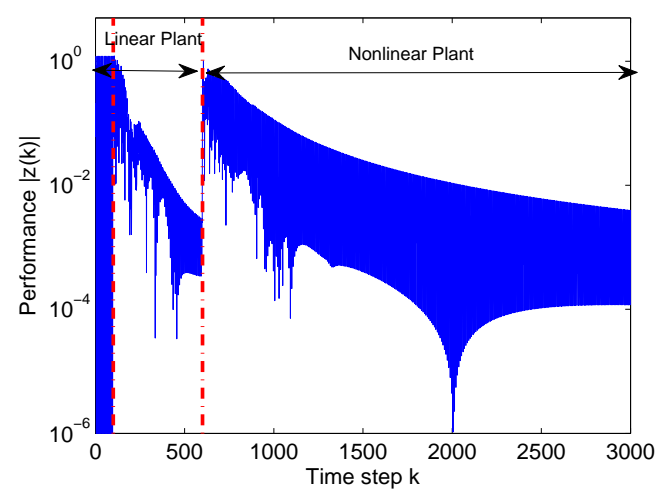

(c)

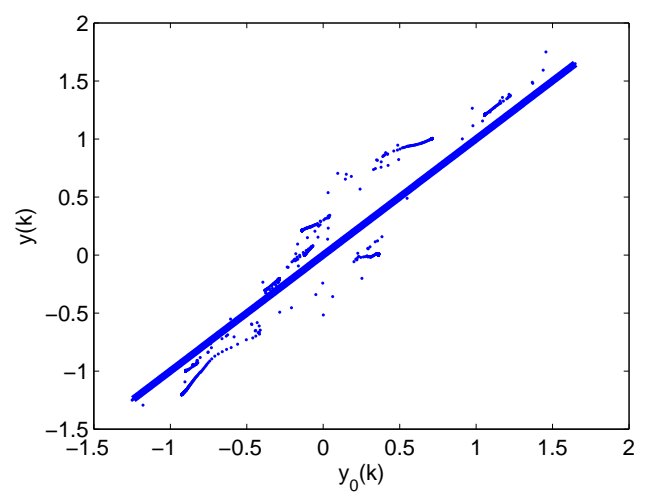

(b)

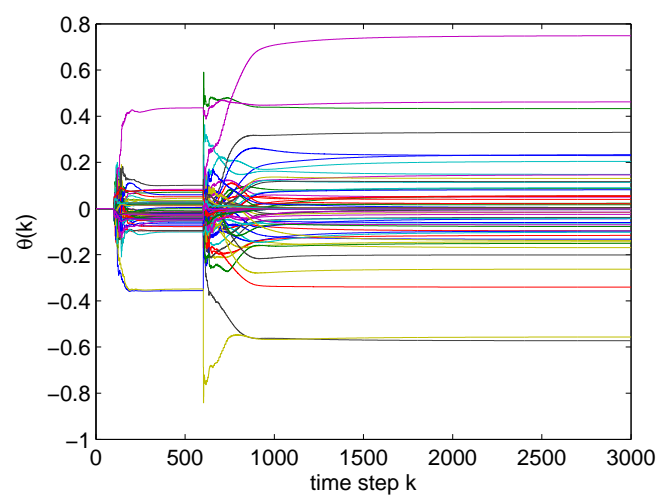

(d)

Figure 8. Example VI.1. (a) shows the hysteretic input nonlinearity $\mathcal{N}_{H}$ given by (44). (b) shows the hysteretic output nonlinearity $\mathcal{N}_{W}$ given by (46). (c) shows the closed-loop response to the command $\operatorname{signal} r(k)=\sin (0.25 \pi k)+0.5 \sin (0.5 \pi k)$ and the disturbance signal $w(k)=0.5 \sin (0.25 \pi k)$ with the linear plant (25) and the nonlinearities of (a) and (b). (d) shows the evolution of the controller gain vector $\theta$.

shows the simulation results. We also consider rate-independent hysteretic input and output nonlinearities. We use the Prandtl-Ishlinskii model (44) with $\eta_{i}=0$ for the input nonlinearity and the Prandtl-Ishlinskii model (46) with $\eta_{i}=0$ for the output nonlinearity. Figure 9 shows the simulation results.

Example VI.2. We consider minimum-phase stable linear plant

$$
G(z)=\frac{z-0.8}{(z+1)(z-0.9)}
$$

and the hysteretic nonlinearities (44) and (46). We use the command signal $r(k)=1$ and the disturbance signal $w(k)=0.4 \sin (0.25 \pi k)$. We let $a=5, n_{\mathrm{c}}=4$, and $P_{0}=I_{3 n_{\mathrm{c}}}$. We turn on the RCAC controller at $k=100$ for the Hammerstein-Wiener system. Figure 10 shows the simulation results. 


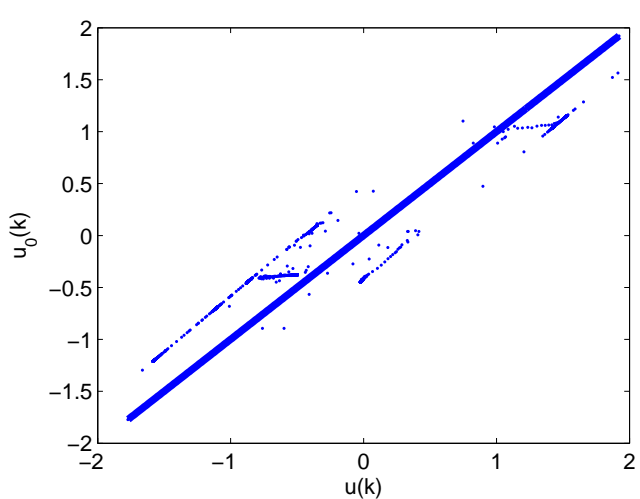

(a)

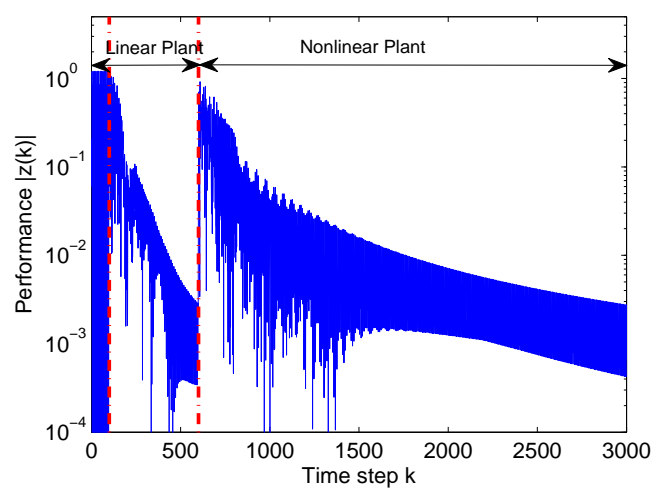

(c)

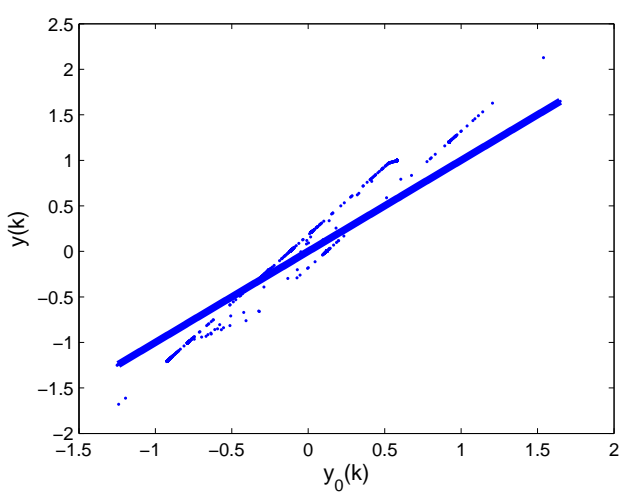

(b)

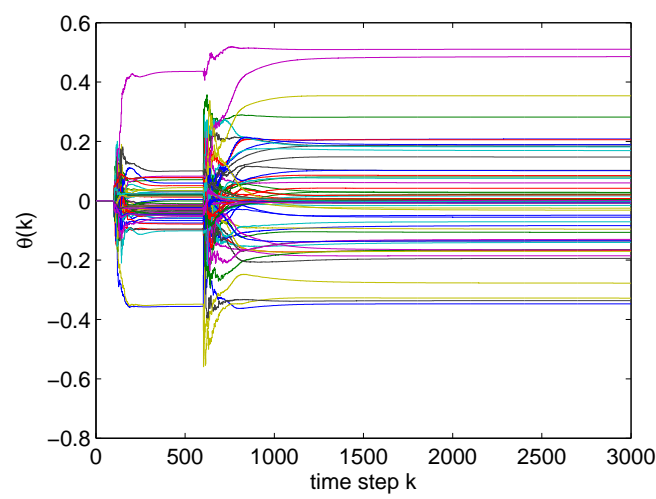

(d)

Figure 9. Example VI.1. (a) shows the hysteretic input nonlinearity $\mathcal{N}_{H}$ given by (44) with $\eta_{i}=0$. (b) shows the hysteretic output nonlinearity $\mathcal{N}_{W}$ given by (46) with $\eta_{i}=0$. (c) shows the closed-loop response to the command signal $r(k)=\sin (0.25 \pi k)+0.5 \sin (0.5 \pi k)$ and the disturbance signal $w(k)=0.5 \sin (0.25 \pi k)$ with the linear plant (25) and the nonlinearities of (a) and (b). (d) shows the evolution of the controller gain vector $\theta$

\section{Numerical Examples: Hysteretic-Memoryless nonlinearities}

Example VII.1. We consider the minimum-phase unstable double integrator plant

$$
G(z)=\frac{z-0.9}{z^{2}-1}
$$

and

$$
\mathcal{N}_{H}(u(k))=\Phi[u](k)
$$

with the saturation output nonlinearity

$$
\mathcal{N}_{W}\left(y_{0}\right)= \begin{cases}-1, & \text { if } y_{0}<-1 \\ y_{0}, & \text { if } 1 \leq y_{0} \leq-1 \\ 1, & \text { if } y_{0}>1\end{cases}
$$

Since these nonlinearities are nondecreasing, we choose $\mathcal{N}_{1}(u)=u$ and $\mathcal{N}_{2}\left(u_{\mathrm{c}}\right)=\operatorname{sat}_{a}\left(u_{\mathrm{c}}\right)$. We let $a=5$, $n_{\mathrm{c}}=23$, and $P_{0}=10.6 I_{3 n_{\mathrm{c}}}$. We use $r(k)=\sin (0.25 \pi k)$ and $w(k)=0.8 \sin (\pi k)+0.5 \sin (0.25 \pi k)$. Figure 11 shows the simulation results. 


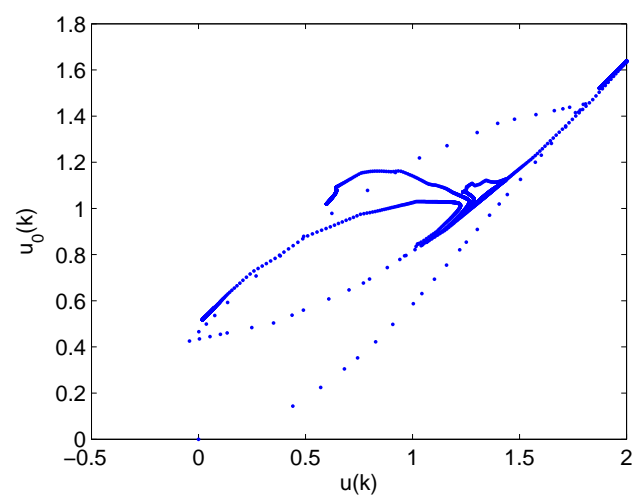

(a)

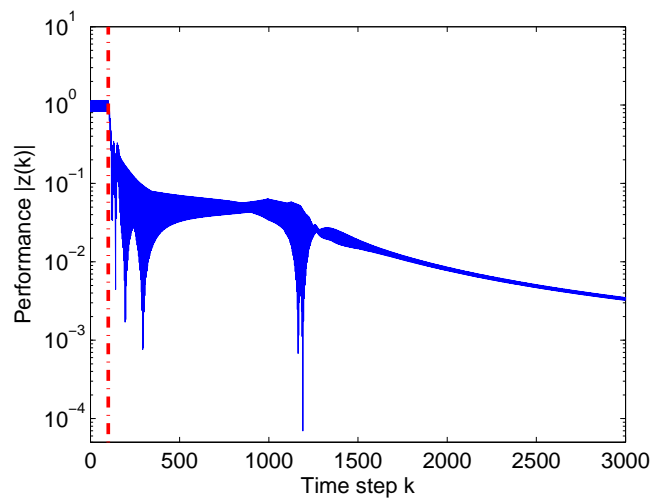

(c)

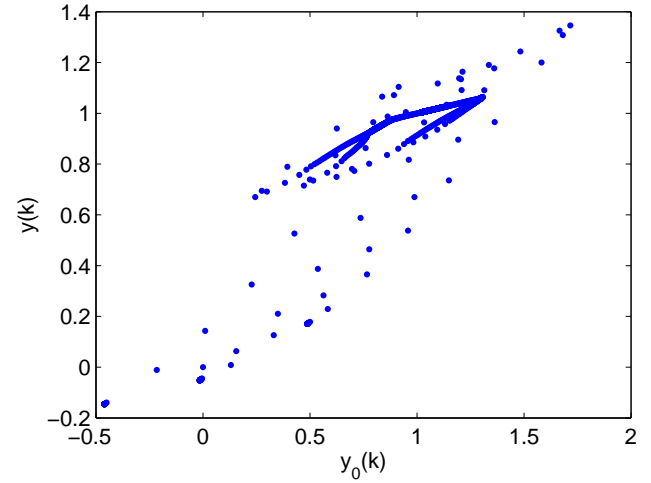

(b)

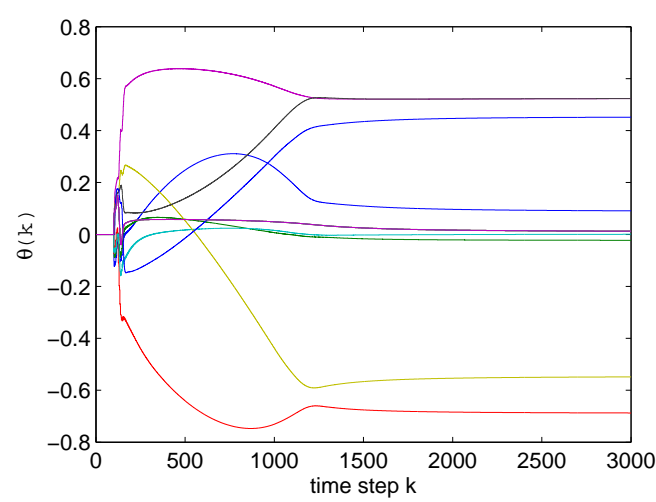

(d)

Figure 10. Example VI.2. (a) shows the rate-dependent hysteretic input nonlinearity $\mathcal{N}_{H}$ given by (44). (b) shows the rate-dependent the hysteretic output nonlinearity $\mathcal{N}_{W}$ given by (46). (c) shows the closed-loop response to the step command signal $r(k)=1$ and the disturbance signal $w(k)=0.4 \sin (0.25 \pi k)$ with the linear plant (48) and the nonlinearities of (a) and (b). (d) shows the evolution of the controller gain vector $\theta$.

Example VII.2. We consider the Lyapunov-stable plant

$$
G(z)=\frac{1}{z-1}
$$

with the input nonlinearity

$$
\mathcal{N}_{H}(u)=-u
$$

and the hysteretic output nonlinearity of the Prandtl-Ishlinskii model

$$
y(k)=\mathcal{N}_{W}\left(y_{0}(k)\right)=\Phi\left[y_{0}\right](k)
$$

The parameters of Prandtl-Ishlinskii model are selected as $n=2, \zeta_{1}=0.5, \zeta_{2}=0.5, \eta_{1}=0.3, \eta_{2}=0.2$, $a_{0}=0.5, a_{1}=0.18, a_{2}=0.162, x_{1}, x_{2}=0$, and $\eta_{i}=0$. Since these nonlinearities are monotonically decreasing, we choose $\mathcal{N}_{1}(u)=-u$ and $\mathcal{N}_{2}\left(u_{\mathrm{c}}\right)=$ sat $_{a}\left(u_{\mathrm{c}}\right)$. We let $a=5, n_{\mathrm{c}}=30$, and $P_{0}=0.1 I_{3 n_{\mathrm{c}}}$. We use $r(k)=\sin (0.25 \pi k)$ and $w(k)=0.1 \sin (0.25 \pi k)$. Figure 19 shows the simulation results.

Example VII.3. We consider the minimum-phase asymptotically stable linear plant

$$
G(z)=\frac{z+0.9}{(z-0.8)(z-0.7)}
$$




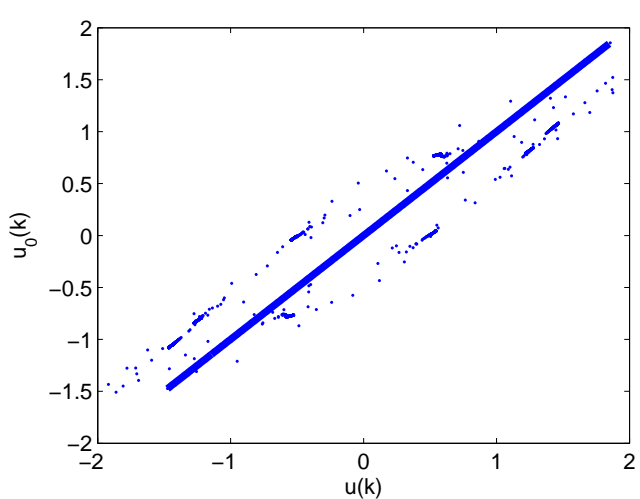

(a)

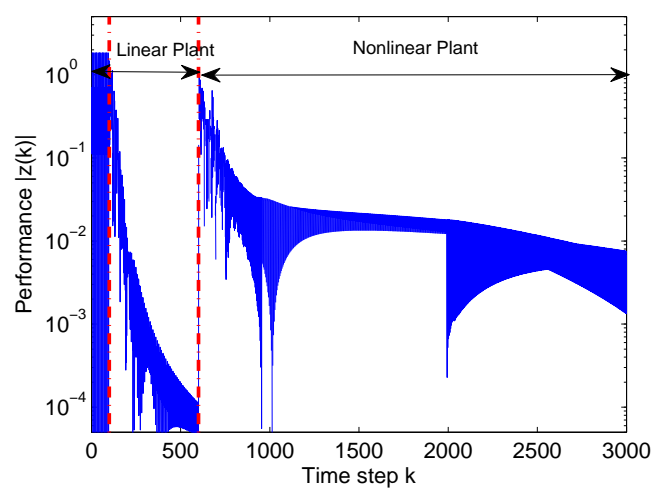

(c)

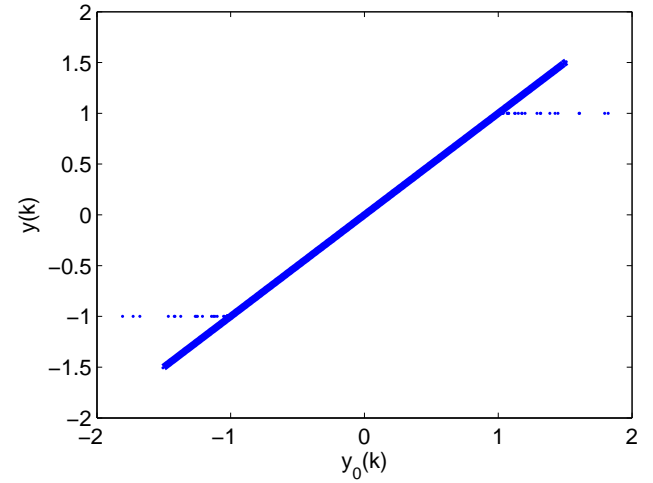

(b)

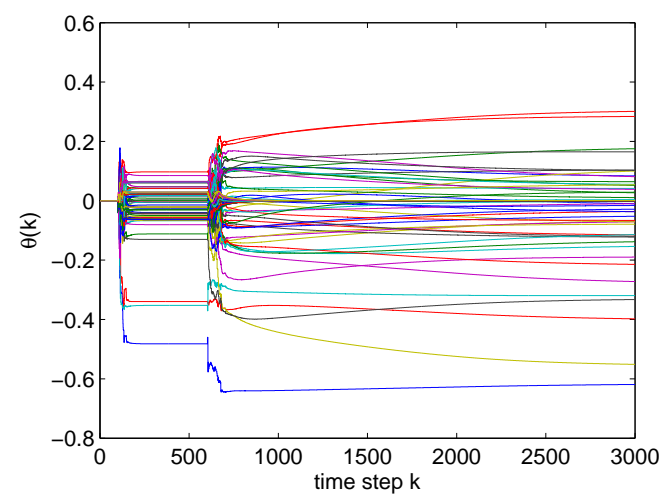

(d)

Figure 11. Example VII.1. (a) shows the rate-dependent hysteretic input nonlinearity $\mathcal{N}_{H}$ given by (57). (b) shows the memoryless output nonlinearity $\mathcal{N}_{W}$ given by (51). (c) shows the closed-loop response to the command $\operatorname{signal} \sin (0.25 \pi k)$ and the disturbance signal $w(k)=0.8 \sin (\pi k)+0.5 \sin (0.25 \pi k)$ with the linear plant (49) and the nonlinearities of (a) and (b). (d) shows the evolution of the controller gain vector $\theta$.

with the deadband input nonlinearity

$$
\mathcal{N}_{H}(u)= \begin{cases}u, & \text { if } u<-0.6 \\ 0, & \text { if }-0.6 \leq u \leq 0.6 \\ u, & \text { if } u>0.6\end{cases}
$$

and the hysteretic output nonlinearity

$$
y(k)=\mathcal{N}_{W}\left(y_{0}(k)\right)=\Phi\left[y_{0}\right](k)
$$

are considered. Since these nonlinearities are nondecreasing, we choose $\mathcal{N}_{1}(u)=u$ and $\mathcal{N}_{2}\left(u_{\mathrm{c}}\right)=\operatorname{sat}_{a}\left(u_{\mathrm{c}}\right)$. We let $a=4, n_{\mathrm{c}}=30$, and $P_{0}=0.1 I_{3 n_{\mathrm{c}}}$. We use $r(k)=\sin (0.25 \pi k)+0.4 \sin (0.5 \pi k)$ and $w(k)=$ $0.5 \sin (0.25 \pi k)+0.2 \sin (\pi k)$. Figure 13 shows the simulation results.

Example VII.4. We consider the minimum-phase asymptotically stable linear plant

$$
G(z)=\frac{z-0.8}{(z-0.9)^{2}}
$$

with the deadband input nonlinearity

$$
\mathcal{N}_{H}(u)= \begin{cases}u, & \text { if } u<-0.4 \\ 0, & \text { if }-0.4 \leq u \leq 0.7 \\ u, & \text { if } u>0.7\end{cases}
$$




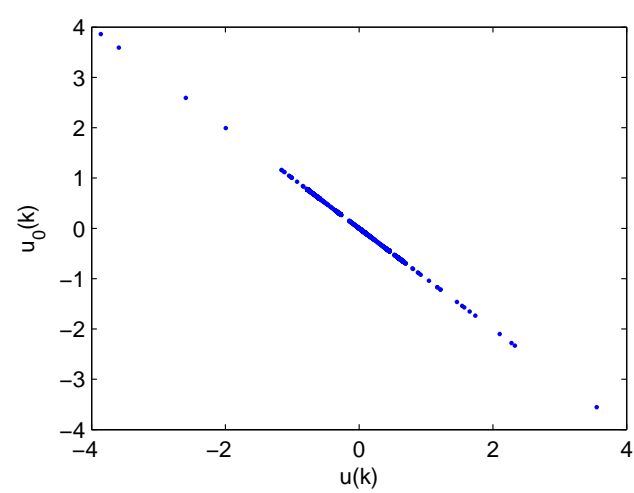

(a)

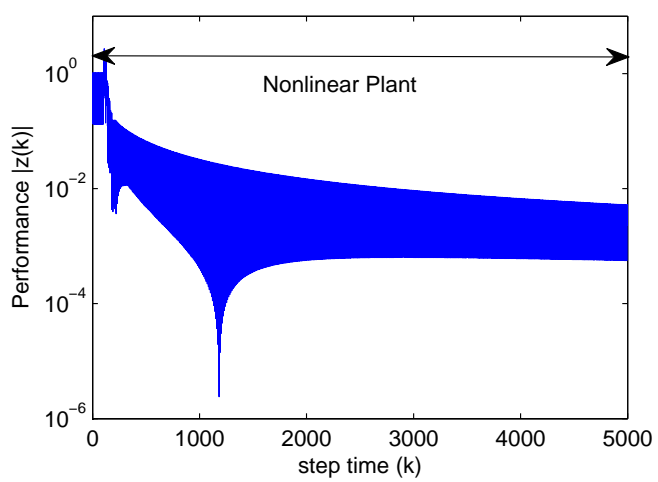

(c)

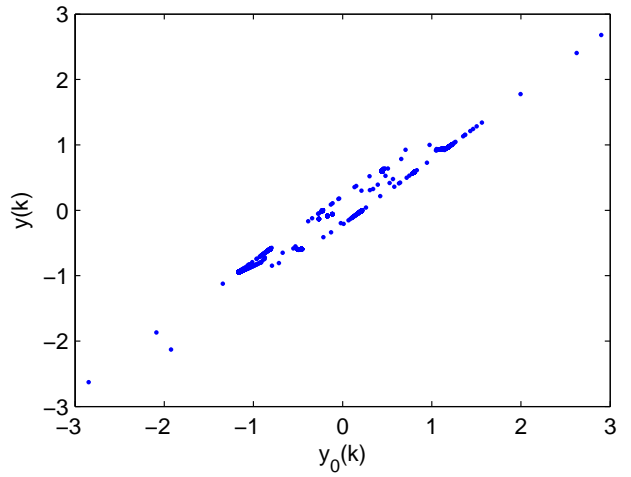

(b)

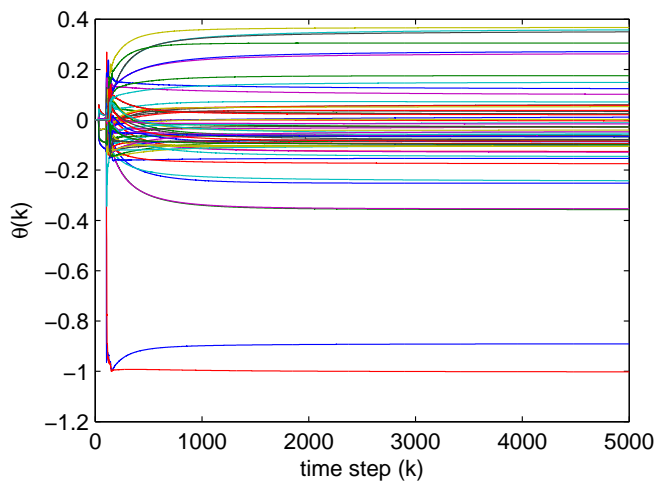

(d)

Figure 12. Example VII.2. (a) shows the memoryless input nonlinearity $\mathcal{N}_{H}$ given by (51), (b) shows the hysteretic output nonlinearity $\mathcal{N}_{W}$ given by (54). (c) shows the closed-loop response to the command $\operatorname{signal} \sin (0.25 \pi k)$ and the disturbance signal $w(k)=0.1 \sin (0.5 \pi k)$ with the linear plant (52) and the nonlinearities of (a) and (b). (d) shows the evolution of the controller gain vector $\theta$.

and the hysteretic output nonlinearity

$$
y(k)=\mathcal{N}_{W}\left(y_{0}(k)\right)=\Phi_{\Gamma}\left[y_{0}\right](k),
$$

where

$$
\Gamma\left(y_{0}\right)=0.9 \tanh \left(1.7 y_{0}-0.1\right)
$$

Since these nonlinearities are nondecreasing, we choose $\mathcal{N}_{1}(u)=u$ and $\mathcal{N}_{2}\left(u_{\mathrm{c}}\right)=\operatorname{sat}_{a}\left(u_{\mathrm{c}}\right)$. We let $a=5$, $n_{\mathrm{c}}=38$, and $P_{0}=2 I_{3 n_{\mathrm{c}}}$. The command signal $r(k)=\sin (0.25 \pi k)$ and the disturbance signal $w(k)=$ $0.5 \sin (0.5 \pi k)+0.3 \sin (\pi k)$ are considered. Figure 14 shows the simulation results.

Example VII.5. We consider the minimum-phase asymptotically stable linear plant

$$
G(z)=\frac{z-0.95}{(z-0.85)(z-0.82)}
$$

with the saturation nonlinearity

$$
v=\mathcal{N}_{H}(u)= \begin{cases}-1, & \text { if } u<-1, \\ u, & \text { if } 1 \leq u \leq-1, \\ 1, & \text { if } u>1,\end{cases}
$$

and the generalized rate-dependent Prandtl-Ishlinskii model

$$
y=\mathcal{N}_{W}\left(y_{0}\right)=\Phi_{\Gamma}\left[y_{0}\right]
$$




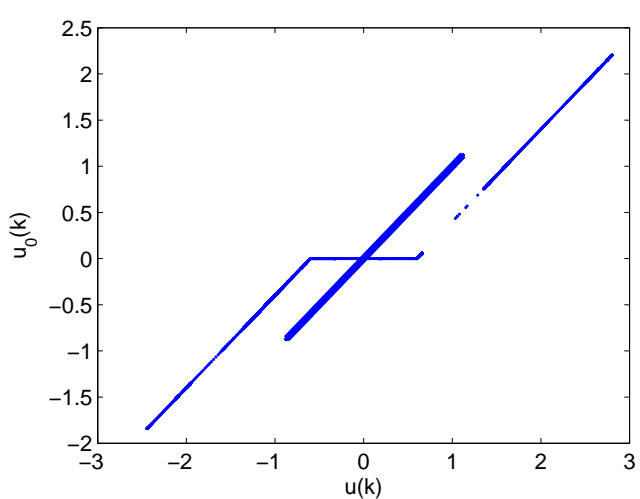

(a)

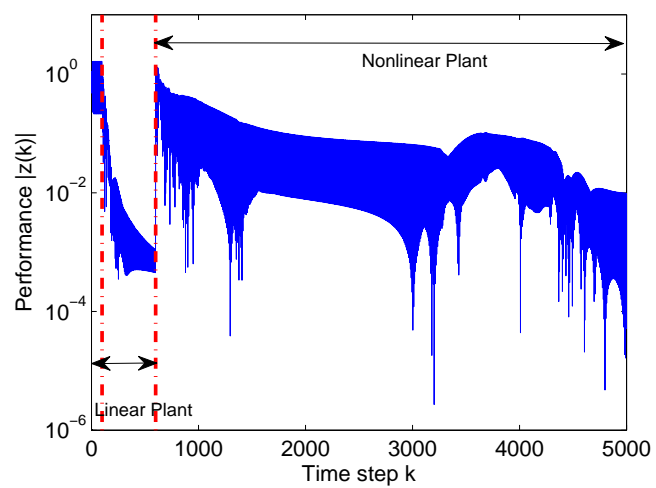

(c)

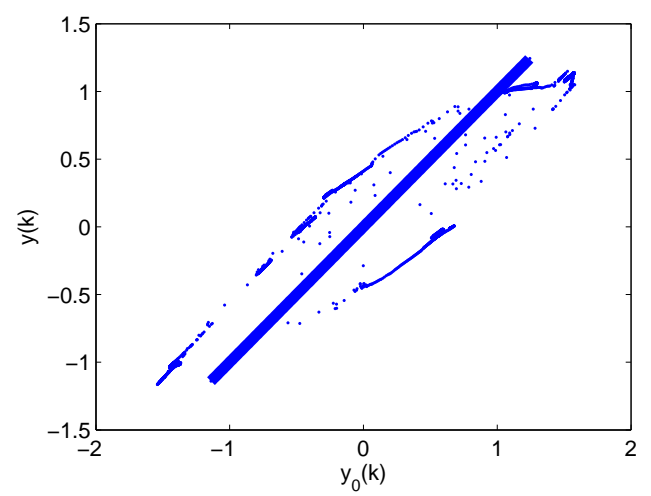

(b)

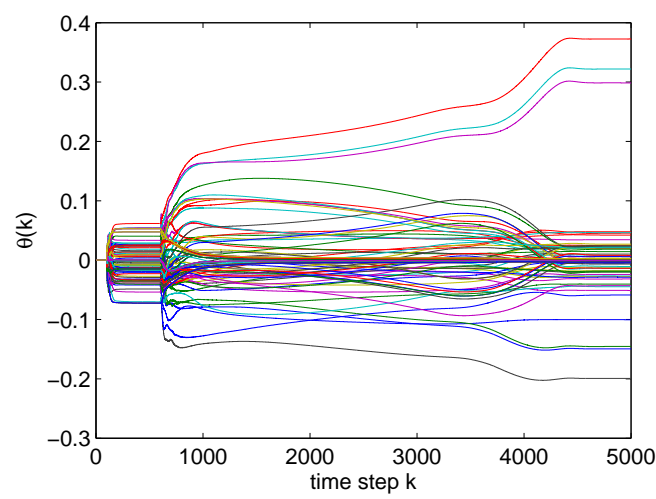

(d)

Figure 13. Example VII.3. (a) shows the memoryless input nonlinearity $\mathcal{N}_{H}$ given by (51), (b) shows the ratedependent hysteretic output nonlinearity $\mathcal{N}_{W}$ given by (57). (c) shows the closed-loop response to the command signal $\sin (0.25 \pi k)+0.4 \sin (0.5 \pi k)$ and the disturbance signal $w(k)=0.5 \sin (0.25 \pi k)+0.2 \sin (\pi k)$ with the linear plant (55) and the nonlinearities of (a) and (b). (d) shows the evolution of the controller gain vector $\theta$.

where

$$
\Gamma\left(y_{0}\right)=0.9 \tanh \left(1.4 y_{0}\right)
$$

Since these nonlinearities are nondecreasing, we choose $\mathcal{N}_{1}(u)=u$ and $\mathcal{N}_{2}\left(u_{\mathrm{c}}\right)=\operatorname{sat}_{a}\left(u_{\mathrm{c}}\right)$. We let $a=5$, $n_{\mathrm{c}}=12$, and $P_{0}=2.7 I_{3 n_{\mathrm{c}}}$. The command signal $r(k)=\sin (0.25 \pi k)$ and the disturbance signal $w(k)=$ $0.5 \sin (0.5 \pi k)+0.3 \sin (\pi k)$ are used. We show the simulation results in Figure 15.

Example VII.6. We consider the asymptotically stable, minimum-phase plant

$$
G(z)=\frac{(z-0.5)(z-0.9)}{(z-0.1)(z-0.5-\jmath 0.5)(z-0.5+\jmath 0.5)},
$$

with the input nonlinearity

$$
\mathcal{N}(u)=\cos (2 u)
$$

and the Prandtl-Ishlinskii model

$$
\mathcal{N}_{W}\left(y_{0}\right)=\Phi\left[y_{0}\right] .
$$

The parameters of the Prandtl-Ishlinskii model are selected as $n=2, \zeta_{1}=0.5, \zeta_{2}=0.5, \eta_{1}=0.3, \eta_{2}=0.2$, $a_{0}=0.5, a_{1}=0.18, a_{2}=0.162, x_{1}, x_{2}=0$, and $\eta_{i}=0$. We use the simulation parameters of Example V.4. Figure 16 shows the simulation results. 


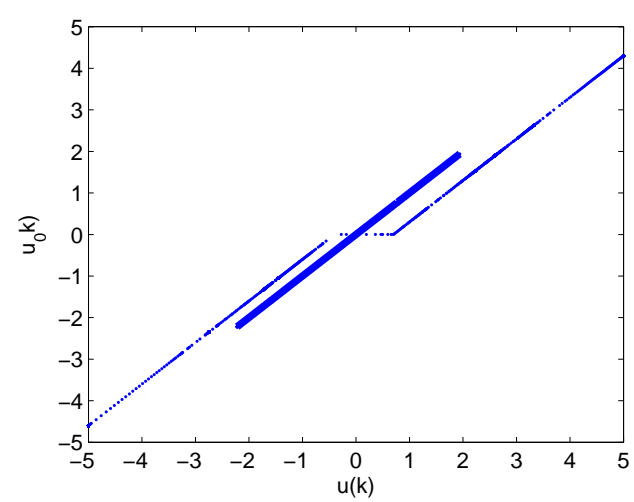

(a)

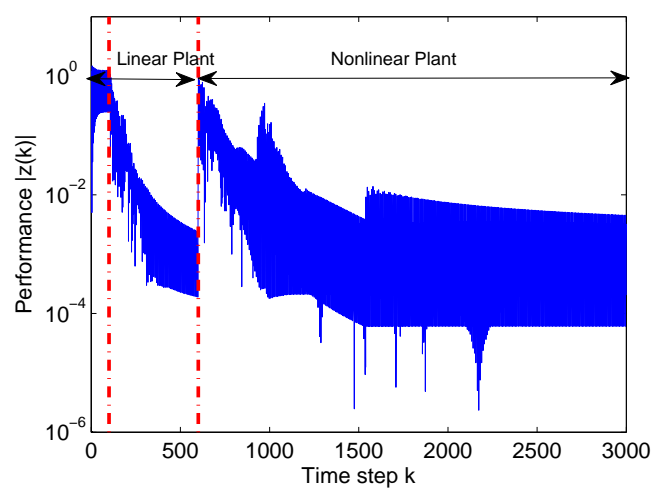

(c)

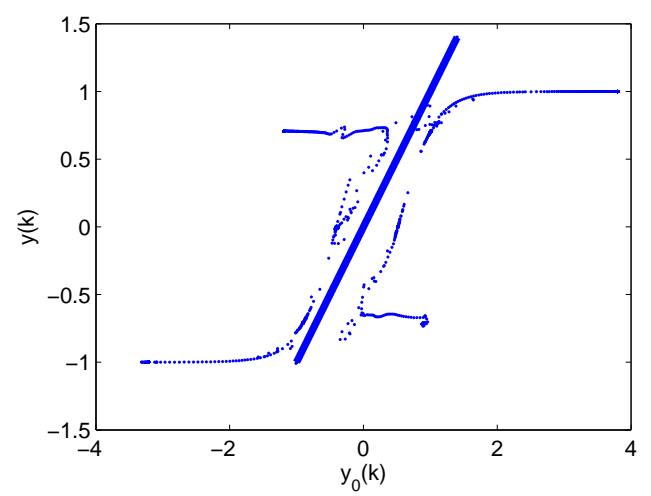

(b)

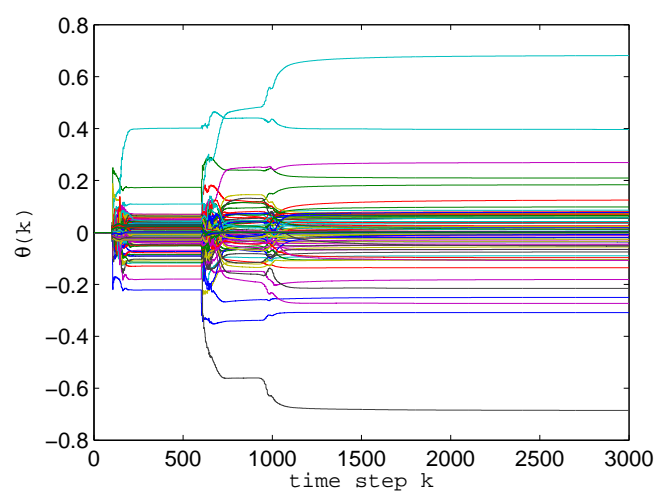

(d)

Figure 14. Example VII.4. (a) shows the deadband input nonlinearity $\mathcal{N}_{H}$ given by (59), (b) shows the rate-dependent hysteretic output nonlinearity $\mathcal{N}_{W}$ given by (60). (c) shows the closed-loop response to the command $\operatorname{signal} \sin (0.25 \pi k)$ and the disturbance signal $w(k)=0.5 \sin (0.5 \pi k)+0.3 \sin (\pi k)$ with the linear plant (58) and the nonlinearities of (a) and (b). (d) shows the evolution of the controller gain vector $\theta$.

Example VII.7. We consider the minimum-phase asymptotically stable linear plant

$$
G(z)=\frac{z-0.8}{(z-0.9)(z+0.9)}
$$

with compound Wiener and Hammerstein nonlinearities defined as

$$
y=\mathcal{N}_{W}\left(y_{0}\right)=\Gamma \mathcal{W}_{1} \circ \Phi \circ \Gamma \mathcal{W}_{2}\left[y_{0}\right]
$$

and

$$
v=\mathcal{N}_{H H}(u)=\Phi \circ \Gamma_{\mathcal{N}_{H}}[u]
$$

where $\Gamma_{\mathcal{W}_{1}}: \mathbb{R} \rightarrow \mathbb{R}, \Gamma_{\mathcal{W}_{2}}: \mathbb{R} \rightarrow \mathbb{R}$, and $\Gamma_{\mathcal{N}_{H H}}: \mathbb{R} \rightarrow \mathbb{R}$ are nondecreasing memoryless nonlinearities. We turn on the RCAC controller at $k=100$ for the compound Hammerstein-Wiener system with

$$
\begin{gathered}
\Gamma_{\mathcal{N}_{H}}[u]= \begin{cases}u, & \text { if } u<-0.1, \\
0, & \text { if }-0.1 \leq u \leq 0.5, \\
u, & \text { if } u>0.3,\end{cases} \\
\Gamma_{\mathcal{W}_{1}}[\cdots]=\tanh (\cdots),
\end{gathered}
$$




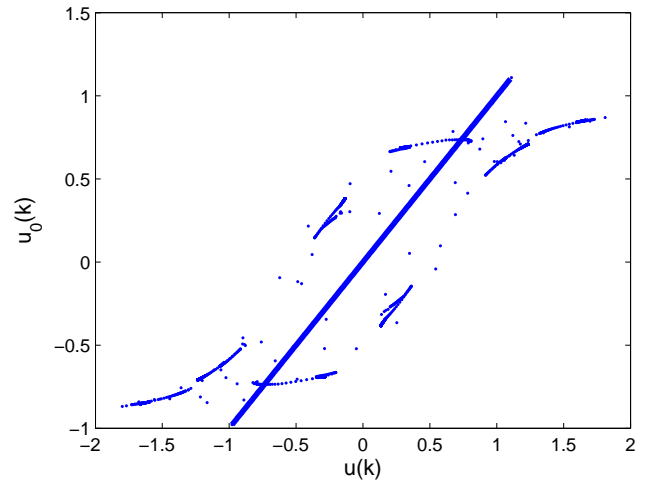

(a)

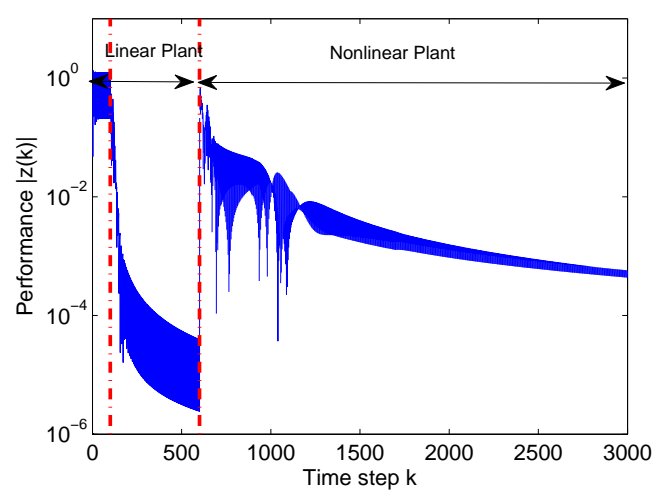

(c)

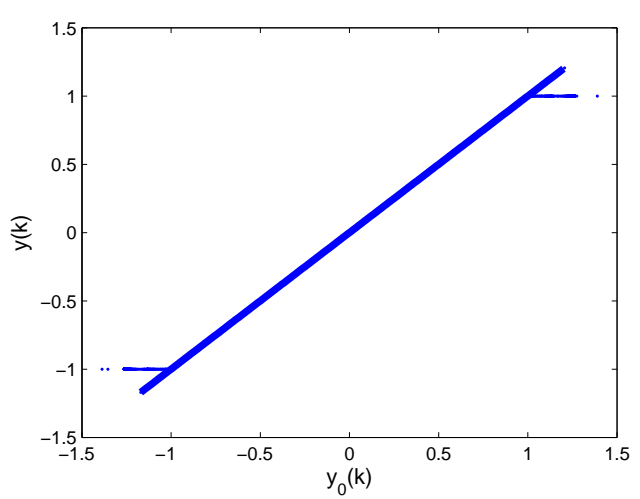

(b)

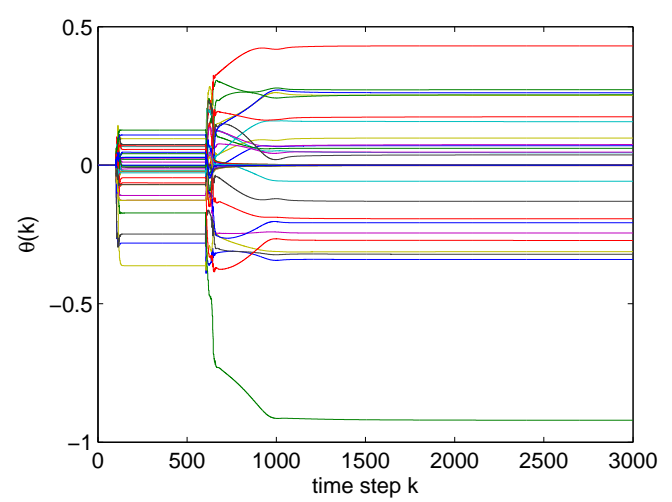

(d)

Figure 15. Example VII.5. (a) shows the memoryless input nonlinearity $\mathcal{N}_{H}$ given by (59), (b) shows the rate-dependent hysteretic output nonlinearity $\mathcal{N}_{W}$ given by (68). (c) shows the closed-loop response to the command signal sin $(0.25 \pi k)$ and the disturbance signal $w(k)=0.5 \sin (0.5 \pi k)+0.3 \sin (\pi k)$ with the linear plant (62) and the nonlinearities of (a) and (b). (d) shows the evolution of the control signal $u(k)$. (e) shows the evolution of the controller gain vector $\theta$. (f) shows the evolution of the output $y(k)$.

and

$$
\Gamma_{\mathcal{W}_{2}}\left[y_{0}\right]= \begin{cases}y_{0}, & \text { if } y_{0}<0 \\ 0, & \text { if } 0 \leq y_{0} \leq 0.4 \\ y_{0}, & \text { if } y_{0}>0.4\end{cases}
$$

We consider the command signal $r(k)=0.8 \sin (0.25 \pi k)+0.2 \sin (0.5 \pi k)$ and the disturbance signal $w(k)=$ $0.5+\sin (\pi k)+0.2 \sin (0.25 \pi k)$. Since these nonlinearities are nondecreasing, we choose $\mathcal{N}_{1}(u)=u$ and $\mathcal{N}_{2}\left(u_{\mathrm{c}}\right)=\operatorname{sat}_{a}\left(u_{\mathrm{c}}\right)$. We let $a=5, n_{\mathrm{c}}=22$, and $P_{0}=I_{3 n_{\mathrm{c}}}$. The simulation results are shown in Figure 17.

Example VII.8. We consider the minimum-phase asymptotically stable linear plant

$$
G(z)=\frac{z-0.7}{(z-0.8)(z-0.6)} .
$$

with the input nonlinearity

$$
\mathcal{N}_{H}(u)=2 u^{3}
$$

and the hysteretic output nonlinearity

$$
y(k)=\mathcal{N}_{W}\left(y_{0}(k)\right)=\Psi_{\zeta_{1}}\left[y_{0}, x_{1}\right](t),
$$




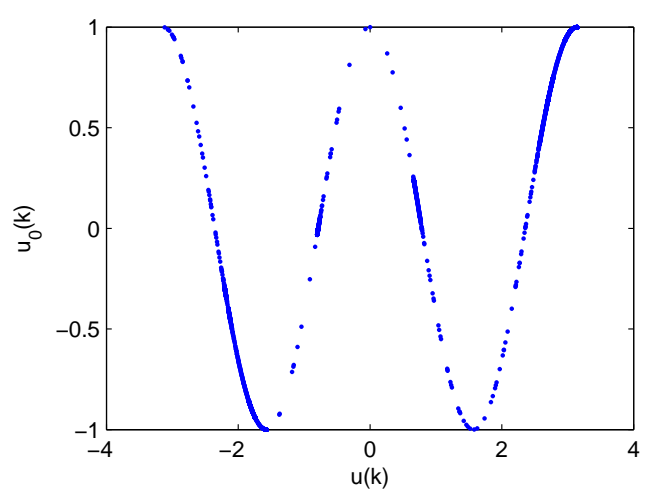

(a)

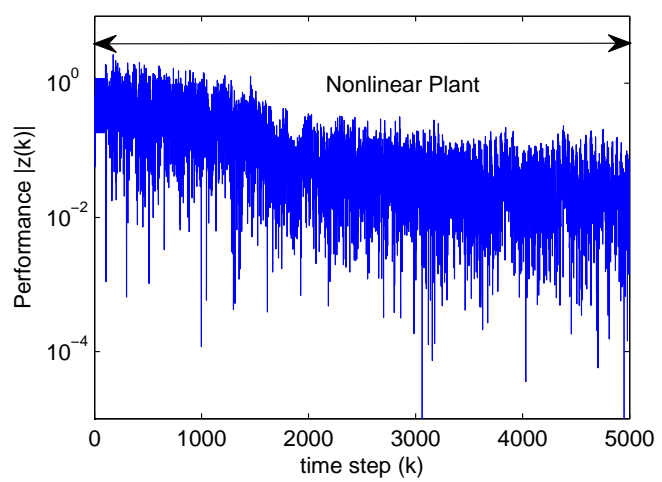

(c)

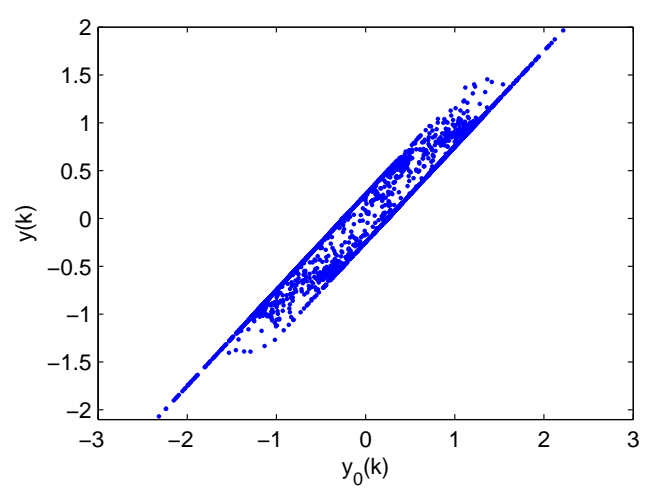

(b)

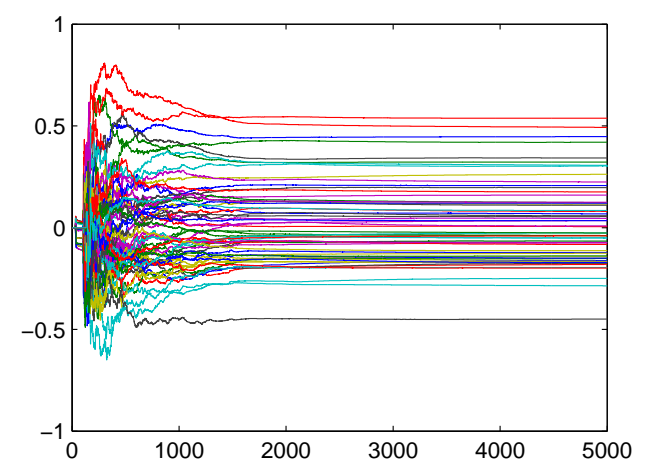

(d)

Figure 16. Example VII.6.(a) shows the memoryless input nonlinearity $\mathcal{N}_{H}$ given by (67). (b) shows the output hysteretic nonlinearity $\mathcal{N}_{W}$ given by (68). (c) shows the closed-loop response to the command $\operatorname{signal} r(k)=1 \sin (0.25 \pi k)$ and the disturbance signal $w(k)=0.25 \sin (0.5 \pi k)$ with the linear plant (66) and the nonlinearities of (a) and (b). (d) shows the evolution of the components of the controller $\theta$.

where $\zeta_{1}=0.1$ and $x_{1}=0$. Since these nonlinearities are nondecreasing, we choose $\mathcal{N}_{1}(u)=u$ and $\mathcal{N}_{2}\left(u_{\mathrm{c}}\right)=\operatorname{sat}_{a}\left(u_{\mathrm{c}}\right)$.We let $a=5, n_{\mathrm{c}}=14$, and $P_{0}=I_{3 n_{\mathrm{c}}}$. We use the command signal $r(k)=0.8$ with two disturbance signals $w(k)=0.5 \sin (0.5 \pi k)$ and $w(k)=0.5$. Figure 18 shows the closed-loop response. The example shows that the RCAC controller drives the performance to arbitrary low levels when a step disturbance signal is applied. However, the performance of the RCAC controller does not achieve these levels when a sinusoidal disturbance signal is used due to distortion from the nonlinearities.

Example VII.9. We consider the minimum-phase asymptotically stable linear plant 75 with the input nonlinearity output nonlinearities of Example VII.8. Since these nonlinearities are nondecreasing, we choose $\mathcal{N}_{1}(u)=u$ and $\mathcal{N}_{2}\left(u_{\mathrm{c}}\right)=\operatorname{sat}_{a}\left(u_{\mathrm{c}}\right)$. We let $a=5, n_{\mathrm{c}}=14$, and $P_{0}=I_{3 n_{\mathrm{c}}}$. We use the command signal $r(k)=1$ with the disturbance signal $w(k)=0.2 \sin (0.5 \pi k)$. In order to show the robustness of the RCAC controller, we present the closed-loop response when $\hat{H}_{d}=2$ and $\hat{H}_{d}=H_{d}=1$. Figure 19 shows the simulation results.

Example VII.10. In this example, we simulate the linear system with memoryless and hysteresis nonlinearities to observe the accuracy of the RCAC controller at various levels of nonlinearity. We consider the unstable minimum-phase plant

$$
G(z)=\frac{z-0.8}{(z-0.7)(z-1.1)}
$$




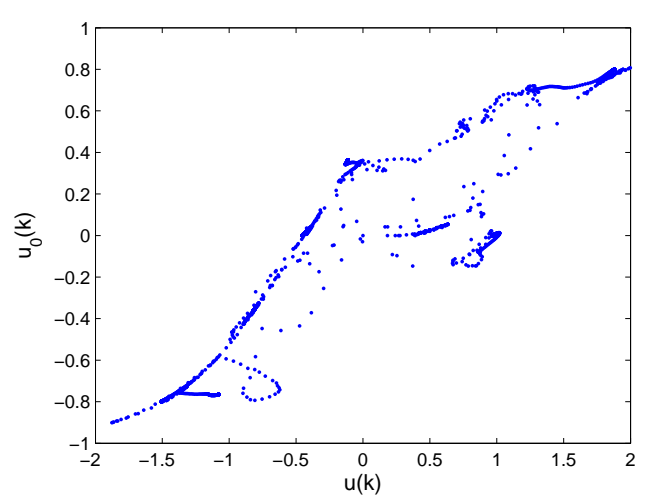

(a)

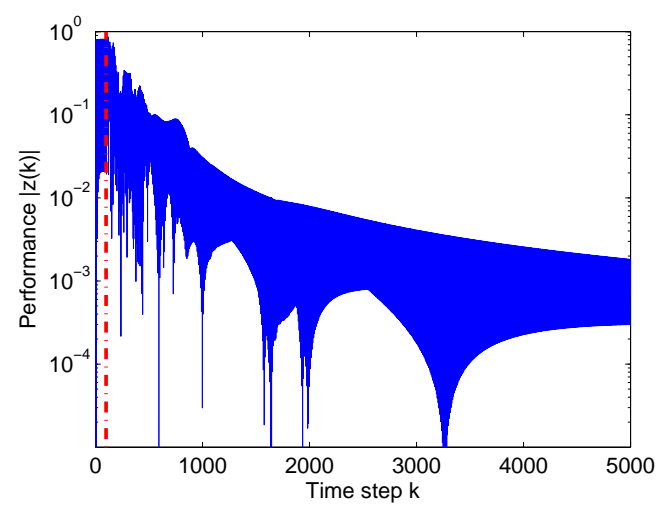

(c)

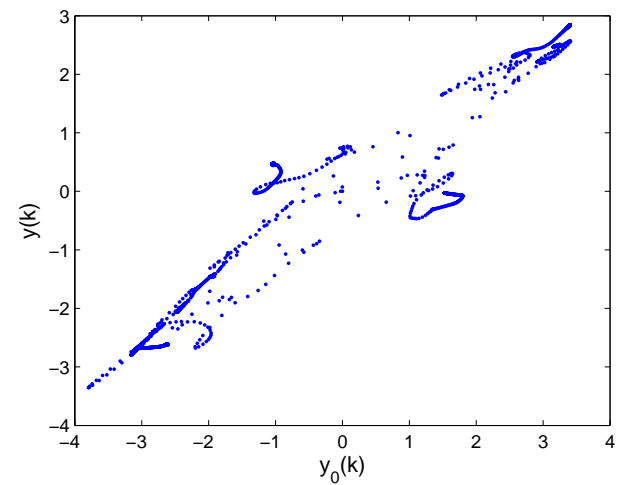

(b)

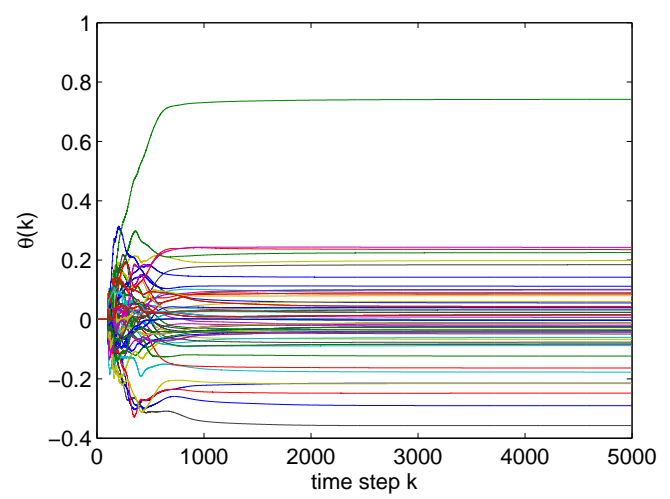

(d)

Figure 17. Example VII.7. (a) shows the input nonlinearity $\mathcal{N}_{H}$ given by (71), (b) shows the output nonlinearity $S N_{W}$ given by (79). (c) shows the closed-loop response to the command signal $r(k)=0.8 \sin (0.25 \pi k)+0.2 \sin (0.5 \pi k)$ and the disturbance signal $w(k)=0.5+\sin (\pi k)+0.2 \sin (0.25 \pi k)$ with the linear plant (69) and the nonlinearities of (a) and (b). (d) shows the evolution of the controller gain vector $\theta$.

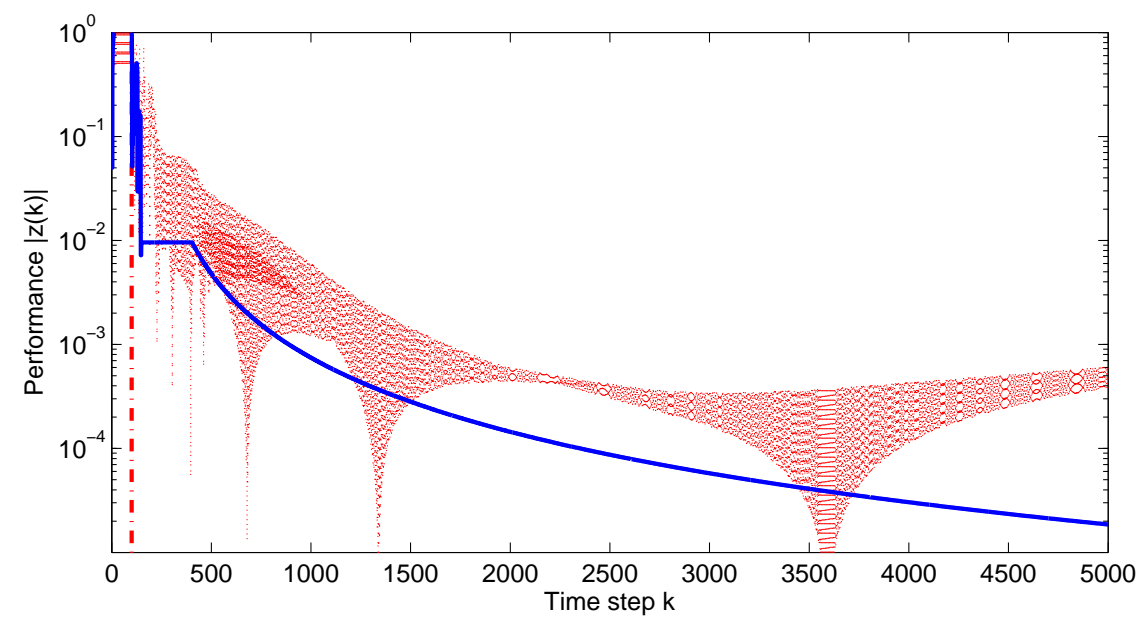

Figure 18. Example VII.8. The closed-loop response to the command signal $r(k)=0.8$ and the nonlinearities (76) and (77) with the linear plant (75) with the disturbance signal $w(k)=0.5$ (solid line), and $w(k)=0.5 \sin (0.5 \pi k)($ dotted line).

with the deadband input nonlinearity

$$
v=\mathcal{N}_{H}(u)=\left\{\begin{array}{l}
u, \quad \text { if } u<-\varrho, \\
0, \quad \text { if }-\varrho \leq u \leq \varrho, \\
u_{\text {of } 2 \text { if } u>\varrho}
\end{array}\right.
$$




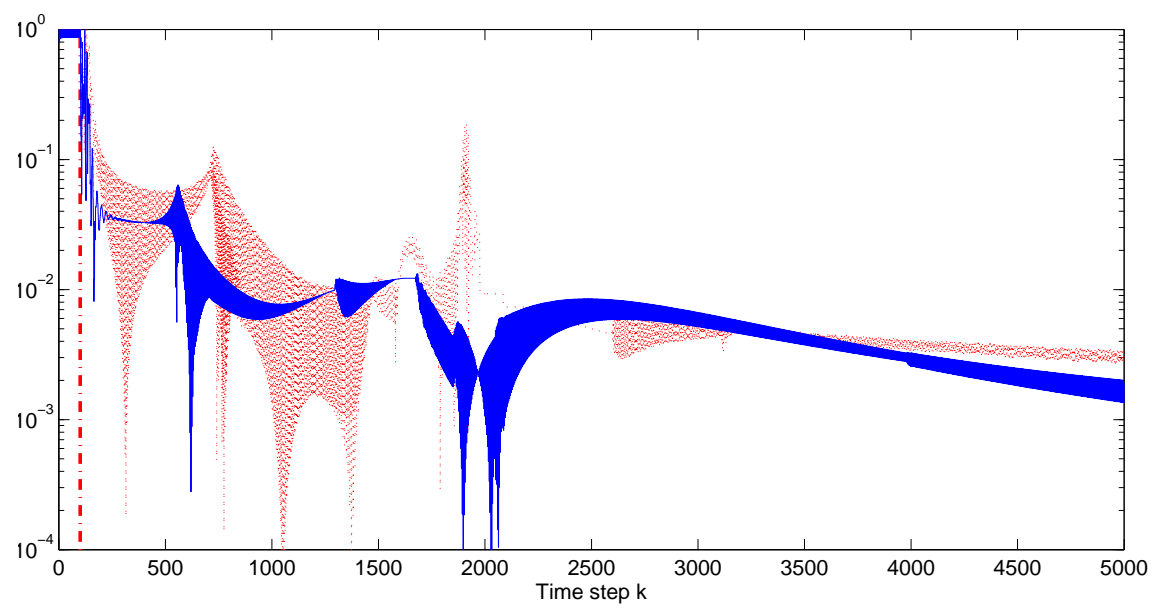

Figure 19. Example VII.9. The closed-loop response to the command signal $r(k)=1$ and the nonlinearities (76) and (77) with the linear plant (75) with the disturbance signal $w(k)=0.2 \sin (0.5 \pi k)$ considering $\hat{H}_{d}=1$ (solid line), and $\hat{H}_{d}=2$ (dotted line).

and the hysteretic output nonlinearity

$$
y(k)=\mathcal{N}_{W}\left(y_{0}(k)\right)=\Psi_{\zeta_{1}}\left[y_{0}, x_{1}\right](t),
$$

where $\varrho$ is a positive constant. We use the command input signal $r(k)=\sin (0.25 k)$ and $x_{1}=0$. Since these nonlinearities are nondecreasing, we choose $\mathcal{N}_{1}(u)=u$ and $\mathcal{N}_{2}\left(u_{\mathrm{c}}\right)=\operatorname{sat}_{a}\left(u_{\mathrm{c}}\right)$. We let $a=5, n_{\mathrm{c}}=15$, and $P_{0}=0.2 I_{3 n_{\mathrm{c}}}$. Figure 20 shows the simulation results for the closed-loop response of the two cases. In the first case we used hysteretic output nonlinearity (80) of $\zeta_{1}=0.2$ and deadband input nonlinearities of $\varrho=0.25$ (solid line) and $\varrho=0.5$ (dotted line). Second case includes deadband input nonlinearity of $\varrho=0.2$ and hysteretic output nonlinearities of $\zeta_{1}=0.25$ (solid line) and $\zeta_{1}=0.5$ (dotted line). Due to the distortion from the nonlinearities, it can be concluded that under sinusoidal command input the RCAC controller does not reduce the error to zero. The example also shows that the accuracy depends on the deadbands in the nonlinearities, where higher deadbands decrease the accuracy of the closed-loop system.

\section{Conclusions}

Retrospective cost adaptive control (RCAC) was applied to a command-following problem for uncertain Hammerstein-Wiener systems with memoryless and hysteretic nonlinearities. RCAC used limited modeling information about the memoryless and hysteretic nonlinearities. RCAC was able to drive the Hammerstein-Wiener system to follow the reference command for linear plants that are asymptotically stable or unstable. However, the accuracy of the RCAC controller depends on the deadband in the nonlinearities and the command input and disturbance signals. Under sinusoidal command input signal or sinusoidal disturbance signal, the RCAC controller does not reduce the error to zero due to the distortion from the nonlinearities. Also, higher deadbands in the Hammerstein-Wiener systems affect the performance of the RCAC controller.

\section{References}

${ }^{1}$ D. Nesic, Controllability for a class of Simple Wiener-Hammerstein Systems, Systems and Control Letters, vol. 36, pp. 51-59, 1999.

${ }^{2}$ D. Nesic and G. Bastin, Stabilizability and Dead-Beat Controllers for Two Classes of Wiener-Hammerstein Models, IEEE Transactions on Automatic Control, vol. 44, pp. 2068-2071, 1999. 


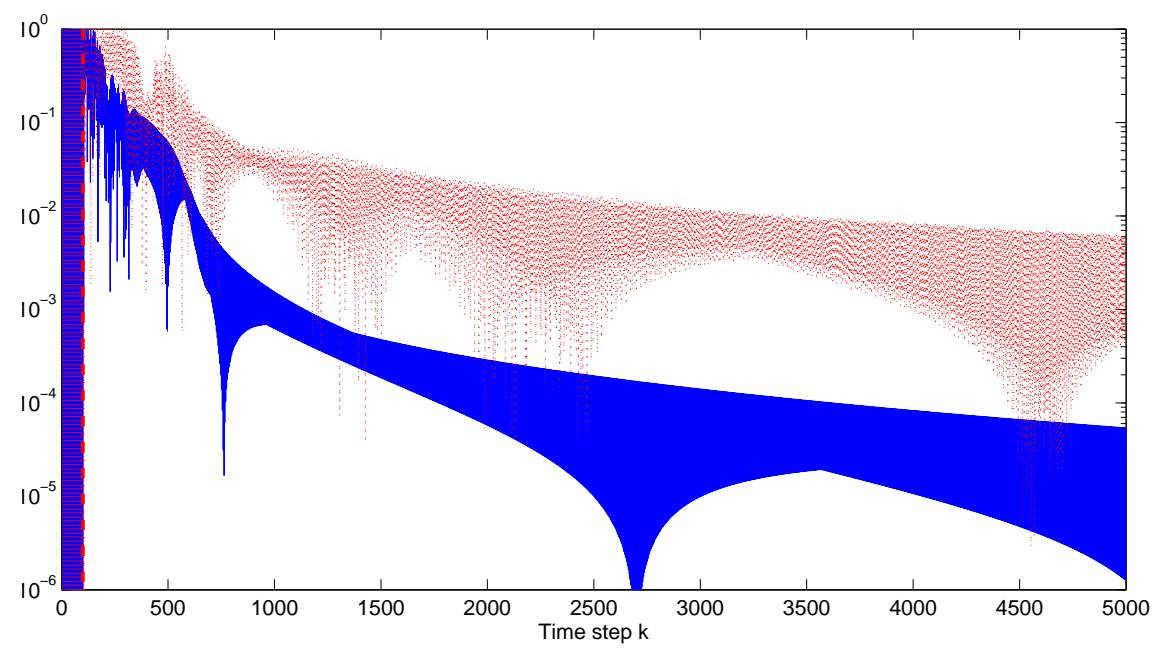

(a)

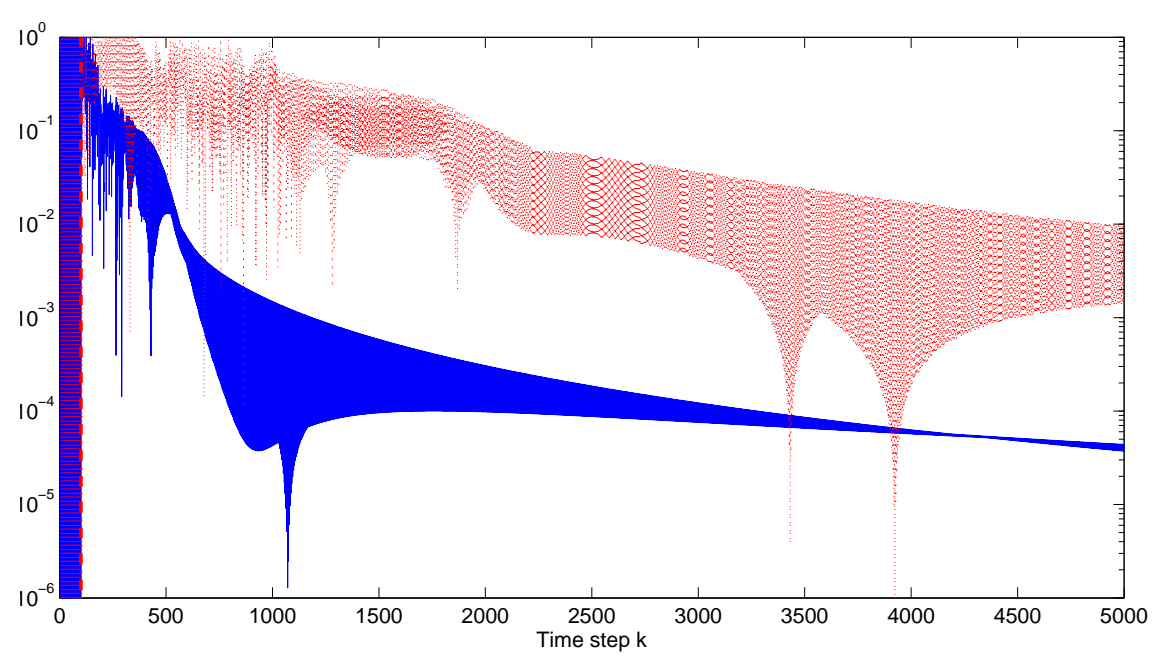

(b)

Figure 20. Example VII.10. The closed-loop response with the command input $r(k)=\sin (0.25 k)$ and the linear plant (78) with the nonlinearities (79) and (80). (a) dotted line: $\zeta_{1}=0.2$ and $\varrho=0.5$, and solid line: $\zeta_{1}=0.2$ and $\varrho=0.25$. (b) dotted line: $\zeta_{1}=0.5$ and $\varrho=0.2$, and solid line: $\zeta_{1}=0.25$ and $\varrho=0.2$.

${ }^{3}$ H. Bloemen, T. Van Den Boom, and H. Verbruggen, Model-based Predictive Control for Hammerstein-Wiener Systems, International Journal of Control, vol. 74, pp. 482-495, 2001.

${ }^{4}$ B. Ding and B. Huang, Output Feedback Model Predictive Control for Nonlinear Systems Represented by HammersteinWiener Model, IET Control Theory Applications, vol. 1, pp. 1302-1301, 2007.

${ }^{5} \mathrm{M}$. Al Janaideh, Y. Feng, S. Rakheja, Y. Tan, and C-Y. Su, Generalized Prandtl-Ishlinskii Hysteresis: Modeling and Robust Control, the 48th IEEE Conference on Decision and Control, Shanghai, China, pp. 7279-7284, 2009.

${ }^{6}$ S. Biagiola, A. Garcia, O. Agamennoni, and J. Figueroa, Robust Control of Wiener Systems: A Case Study, the 14th Mediterranean Conference on Control and Automation, Ancona, Italy, pp. 1-6, 2006.

${ }^{7}$ G. Tao, Adaptive Control of Systems with Actuator and Sensor Nonlinearities, Wiley, 1996.

${ }^{8}$ G. Pajunen, Adaptive control of Wiener type nonlinear systems, Automatica, vol. 28, pp. 781-785, 1992.

${ }^{9}$ J. B. Hoagg, M. A. Santillo, and D. S. Bernstein, Discrete-Time Adaptive Command Following and Disturbance Rejection for Minimum Phase Systems with Unknown Exogenous Dynamics, IEEE Transactions on Automatic Control, vol. 53, pp. 912-928, 2008.

${ }^{10}$ M. A. Santillo and D. S. Bernstein, Adaptive Control Based on Retrospective Cost Optimization, AIAA Journal of Guidance, Control, and Dynamics, vol. 33, pp. 289-304, 2010. 
${ }^{11}$ A. M. D'Amato, E. D. Sumer, and D. S. Bernstein, Retrospective Cost Adaptive Control for Systems with Unknown Nonminimum-Phase Zeros, AIAA Guidance, Navigation, and Control Conference, Portland, OR, August 2011, AIAA-20116203.

${ }^{12}$ A. M. D'Amato, E. D. Sumer, K. S. Mitchell, A. V. Morozov, J. B. Hoagg, and D. S. Bernstein, Adaptive Output Feedback Control of the NASA GTM Model with Unknown Nonminimum-Phase Zeros, AIAA Guidance, Navigation, and Control Conference, Portland, OR, August 2011, AIAA-2011-6204.

${ }^{13}$ J. Yan, A. M. D'Amato, D. Sumer, J. B. Hoagg, and D. S. Bernstein, Adaptive Control of Uncertain Hammerstein Systems Using Auxiliary Nonlinearities, Proceedings of the 51st IEEE Conference on Decision and Control, Maui, HI, December 2012.

${ }^{14}$ A. M. D'Amato, E. D. Sumer, and D. S. Bernstein, Frequency-Domain Stability Analysis of Retrospective-Cost Adaptive Control for Systems with Unknown Nonminimum-Phase Zeros, Proceedings of IEEE Conference on Decision and Control, pp. 1098-1103, Orlando, FL, December 2011.

${ }^{15}$ M. Al Janaideh, D. Sumer, J. Yan, A. D’Amato, B. Drincic, K. Aljanaideh, and D. S. Bernstein, Adaptive Control of Uncertain Linear Systems with Uncertain Hysteretic Input Nonlinearities, Proceedings of the 2012 ASME Dynamic Systems and Control Conference, Lauderdale, FL, October 2012.

${ }^{16}$ J. Yan, A. M. D'Amato, and D. S. Bernstein, Adaptive NARMAX Retrospective Cost Control of Wiener-Hammerstein Systems, AIAA Guidance, Navigation, and Control Conference, Minneapolis, MN, August 2012.

${ }^{17}$ M. Al Janaideh, S. Rakheja, and C-Y. Su, Experimental Characterization and Modeling of Rate-Dependent Hysteresis of a Piezoceramic Actuator, Mechatronics, vol. 19, pp. 656-670, 2009.

${ }^{18}$ P. Krejčí, M. Al Janaideh, and F. Deasy, Inversion of Hysteresis and Creep Operators, Physica B, vol. 407, pp. 1354-1356, 2012.

${ }^{19}$ K. K. Leang, Q. Zou, and S. Devasia, Feedforward Control of Piezoactuators in Atomic Force Microscope Systems, IEEE Control Systems Magazine, vol. 29, pp. 70-82, 2009. 